\title{
Systematic Identification of Anti-Fungal Drug Targets by a Metabolic Network Approach
}

\begin{abstract}
Martin Kaltdorf ${ }^{1 \dagger}$, Mugdha Srivastava ${ }^{1 \dagger}$, Shishir K. Gupta ${ }^{1 \dagger}$, Chunguang Liang ${ }^{1 \dagger}$, Jasmin Binder ${ }^{2}$, Anna-Maria Dietl ${ }^{3}$, Zohar Meir ${ }^{4}$, Hubertus Haas ${ }^{3}$, Nir Osherov ${ }^{4}$, Sven Krappmann ${ }^{2}$ and Thomas Dandekar ${ }^{1 *}$
\end{abstract}

\begin{abstract}
${ }^{1}$ Department of Bioinformatics, Biocenter, University of Würzburg, Würzburg, Germany, ${ }^{2}$ Microbiology Institute - Clinical Microbiology, Immunology and Hygiene, Friedrich-Alexander University Erlangen-Nürnberg, University Hospital of Erlangen, Erlangen, Germany, ${ }^{3}$ Division of Molecular Biology/Biocenter, Medical University Innsbruck, Innsbruck, Austria, ${ }^{4}$ Aspergillus and Antifungal Research Laboratory, Department of Clinical Microbiology and Immunology, Sackler School of Medicine, Tel Aviv University, Tel-Aviv, Israel
\end{abstract}

\section{OPEN ACCESS}

Edited by:

Thomas Pfeiffer,

Massey University, New Zealand

Reviewed by:

Bas Teusink,

Vrije Universiteit Amsterdam,

Netherlands

Ines Heiland,

UiT The Arctic University of Norway,

Norway

*Correspondence:

Thomas Dandekar

dandekar@

biozentrum.uni-wuerzburg.de

${ }^{\dagger}$ These authors have contributed equally to this work.

Specialty section:

This article was submitted to Metabolomics

a section of the journal

Frontiers in Molecular Biosciences

Received: 31 December 2015

Accepted: 24 May 2016

Published: 17 June 2016

Citation:

Kaltdorf M, Srivastava M, Gupta SK Liang C, Binder J, Dietl A-M, Meir Z, Haas H, Osherov N, Krappmann S and Dandekar T (2016) Systematic Identification of Anti-Fungal Drug Targets by a Metabolic Network Approach. Front. Mol. Biosci. 3:22. doi: 10.3389/fmolb.2016.00022
New antimycotic drugs are challenging to find, as potential target proteins may have close human orthologs. We here focus on identifying metabolic targets that are critical for fungal growth and have minimal similarity to targets among human proteins. We compare and combine here: (I) direct metabolic network modeling using elementary mode analysis and flux estimates approximations using expression data, (II) targeting metabolic genes by transcriptome analysis of condition-specific highly expressed enzymes, and (III) analysis of enzyme structure, enzyme interconnectedness ("hubs"), and identification of pathogen-specific enzymes using orthology relations. We have identified 64 targets including metabolic enzymes involved in vitamin synthesis, lipid, and amino acid biosynthesis including 18 targets validated from the literature, two validated and five currently examined in own genetic experiments, and 38 further promising novel target proteins which are non-orthologous to human proteins, involved in metabolism and are highly ranked drug targets from these pipelines.

Keywords: metabolism, targets, antimycotics, modeling, structure, interaction, fungicide

\section{INTRODUCTION}

The treatment of invasive fungal infections caused by the versatile saprophytic fungus Aspergillus fumigatus is challenging (Denning, 1998). While the healthy human immune system is able to fend off A. fumigatus infections in general, immune-deficient patients are highly vulnerable against invasive aspergillosis. Aspergillosis is one of the major lethal conditions in immunocompromised patients (Dagenais and Keller, 2009). In eukaryotic pathogens, most potential protein targets for antimycotic development bear a considerable risk of toxic side effects for the patient as a similar protein might be present in the human host.

Although several anti-mycotic strategies exist, they are only partially effective due to the significant immunosuppression of those patients. Therefore, the development of new therapeutic strategies against $A$. fumigatus infection is crucial.

Targeting the metabolism of pathogens is in general a valid strategy as it is central for pathogen survival and there is also a lower chance for development of resistance mutations as those usually affect fitness and are thus counter selected (Kohanski et al., 2010). 
Unlike many other approaches that exploit a direct antifungal therapy pursuing identified antimycotic leads, we want to introduce here a novel, general strategy to tackle a pathogen at the metabolic level, choosing the human-pathogenic mold A. fumigatus as example. Known challenges in the search for new antimycotic targets include the high similarity between fungal genes and those of the human host. To minimize this problem, we combine three different bioinformatics approaches that we have previously developed to target the pathogen's primary metabolism: (I) metabolic modeling (for instance applied to $S$. aureus antibiotics in Cecil et al., 2015): direct metabolic network modeling using elementary mode analysis and flux estimates constrained by applying gene expression data, (II) enzyme regulation-based strategy: targeting metabolic genes by transcriptome analysis of conditionspecific highly expressed enzymes (for instance applied to $S$. aureus antibiotics in Cecil et al., 2011), (III) protein-protein interaction-based strategy: analysis of enzyme structure, enzyme interconnectedness ("hubs") and identification of pathogenspecific enzymes using orthology relations (for instance applied in viral infections in Shityakov et al., 2015).

Each of these approaches has its strengths and limitations, however, their combination offers a powerful tool to reveal metabolic targets for later drug development. Based on the resulting candidates we suggest a prioritized list of target genes that are important for A. fumigatus but have no close orthologs in humans. By focusing the effort on the metabolic pathways for (a) vitamin synthesis, (b) lipid biosynthesis, and (c) amino acids biosynthesis, we developed a pipeline that integrates and compares results from all three bioinformatics approaches (IIII) to reduce and focus the target list to the most promising candidate genes.

These candidate proteins for targeting fungal metabolism by antimycotics were in part validated according to literature evidence, several are currently tested and evaluated experimentally while others are still available for targeting. Additional information can be incorporated for further refinement and iterations of our combined target screening pipeline. Our workflow is not restricted to A. fumigatus but can also be easily transferred to other pathogens which are similar challenging to target.

\section{MATERIALS AND METHODS}

\section{Metabolic Modeling}

Figure 1 shows the metabolic network modeling approach applied as a first strategy to target fungal metabolism by interfering substances. We outline the flow chart of analysis procedures used to obtain a metabolic network which can be used for prediction of flux and elementary modes with the help of various different types of data and software.

As a first step we apply a process of pathway reconstruction that identifies the $A$. fumigatus enzymes involved in the different pathways.: For this we used biochemical databanks such as KEGG (Kanehisa and Goto, 2000; Kanehisa et al., 2014), Roche pathways (Michal and Schomburg, 2012), and Metacyc (Caspi et al., 2016). Sometimes data from this source are not complete or incorrectly annotated. Such knowledge gaps were filled by literature and expert knowledge including sequence analysis and reannotation of incorrect annotations. The complete primary metabolism from $A$. fumigatus was modeled in this way to yield a metabolic network, including the major carbohydrate metabolism (glycolysis, pentose phosphate pathway, TCA cycle), nucleotide biosynthesis, amino acid biosynthesis and degradation, and fermentation pathways.

Furthermore, cofactors and cell wall synthesis were also taken into account. Moreover, we created sub-networks for vitamins and fatty acid metabolism. For these additional components we looked only at selective reactions and pathways, which we considered particularly promising for targeting and being absent in humans. Accordingly, we selected basic fatty acid metabolism, ergosterol, and glucan metabolism, as well as vitamin metabolism with focus on riboflavin and thiamine.

\section{Elementary Mode Analysis}

A method to identify metabolic pathways that might be crucial for growth is the elementary mode analysis. A flux mode is a set of enzymes, which balance all metabolites within a metabolic network such that these "internal metabolites" do not accumulate or are diminished (not considering sources and drains, the "external metabolites"). Those flux modes (combinations of enzymes) which cannot be decomposed further without affecting this balance are called elementary modes. We computed these elementary modes using the Metatool program (von Kamp and Schuster, 2006), which has been integrated within the YANA software package (Schwarz et al., 2005). Further analysis of the calculated pathways (i.e., all elementary modes) considered which metabolic enzymes are valid antibiotic targets. These are enzymes that are essential for the metabolism as without their operation there are no alternative routes available to produce critical metabolites required for growth.

\section{Flux Mode Strength}

To calculate condition-specific strengths of different metabolic fluxes, large-scale transcriptome data-sets were used as constraints to fit the metabolic model with the aim to estimate flux distributions (Schwarz et al., 2005, 2007) for optimal growth conditions and changes under biofilm condition. The training procedure and algorithms involved (Gradient descent: BFGSBoyden-Fletcher-Goldfarb-Shannon optimization method) have been described previously (Liang et al., 2011).

In summary, we use two methods sequentially: First, elementary mode analysis provides an overview of all metabolic pathways accessible for the pathogen. Second, based on this network, algorithms such as YANA, YANAsquare, and YANAvergence offer various routines to calculate implied flux values for different pathways using gene or protein expression data. Changes in metabolite concentration are best to calculate by flux analysis, but being elaborate to measure and hence seldom available, they were therefore not available here. Both methods identify those metabolic enzymes which are essential for growth, either as they are involved in unique routes to provide metabolites required for growth (elementary mode analysis) 


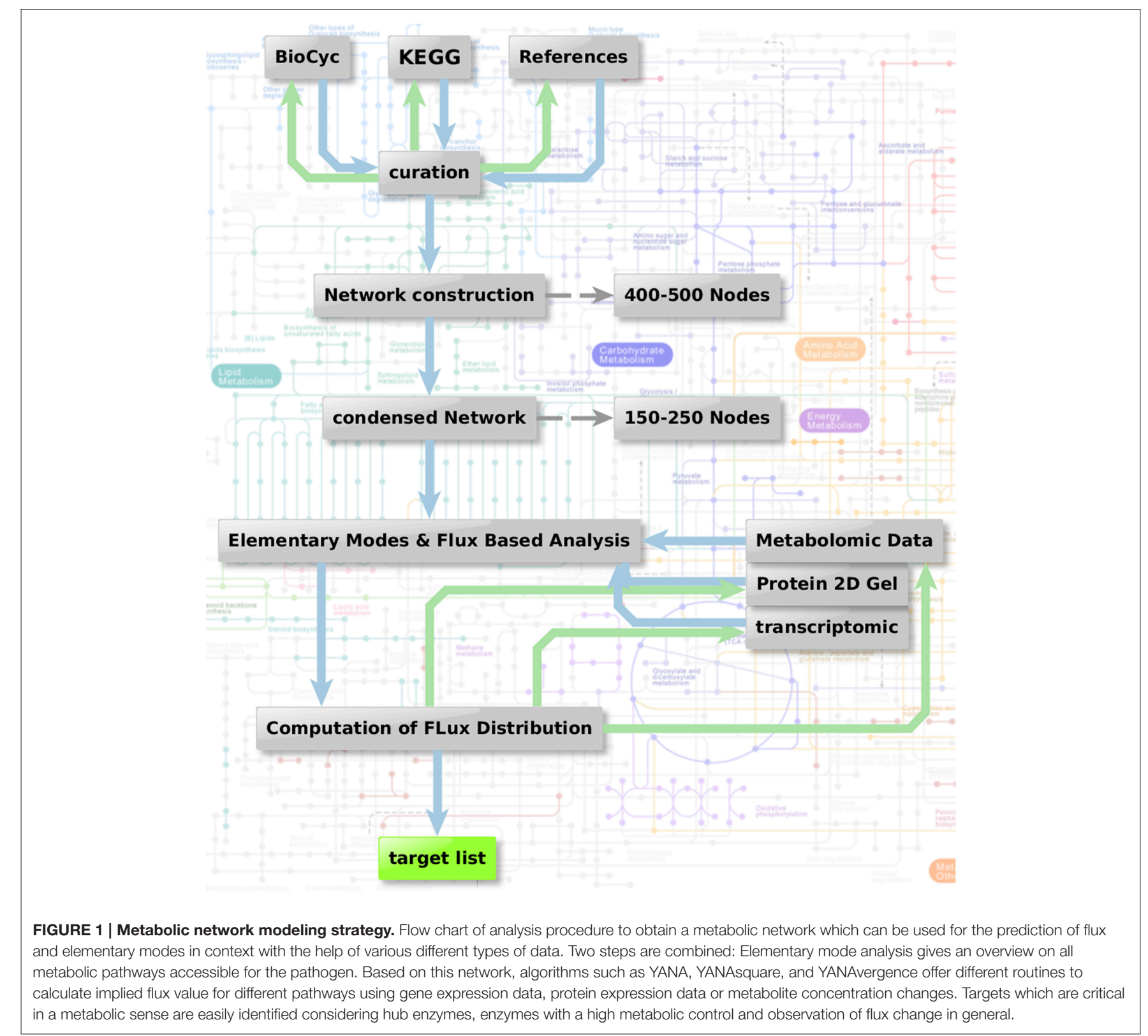

or as they carry a strong metabolic flux, either constantly (housekeeping enzymes) or in the relevant situation of invasion and infection.

\section{Enzyme Regulation-Based Strategy}

Figure 2 depicts the flow chart of the enzyme regulation-based strategy. Transcriptome datasets we used for our comparison analysis involved: Bruns et al. (2010) (accession GSE19430); Schrettl et al. (2010) (accession GSE22052); Willger et al. (2008) (accession GSE12376). These data sets were available from Gene Expression Omnibus (Edgar et al., 2002; Barrett et al., 2013), a database collection of extensive experimental gene expression results as well as from additional experimental sources (McDonagh et al., 2008). We analyzed the datasets using the
GEO2R framework, a built-in function of the GEO database, which uses the R statistics software (Davis and Meltzer, 2007; Huber et al., 2015; R Core Team, 2015; Ritchie et al., 2015) for normalization, fitting, comparison, and visualization of provided microarray data and calculates the resulting differential expressed genes.

After identification of relevant experiments to reveal key enzymes involved in metabolic adaptation as prospective antimycotic targets we defined two different groups of samples (background and relevant condition).

Relevant experiments concerned here available GEO datasets on A. fumigatus genes involved in virulence. Such genes have to be induced by appropriate conditions, we found the following datasets: changes during invasion initiation (McDonagh et al., 


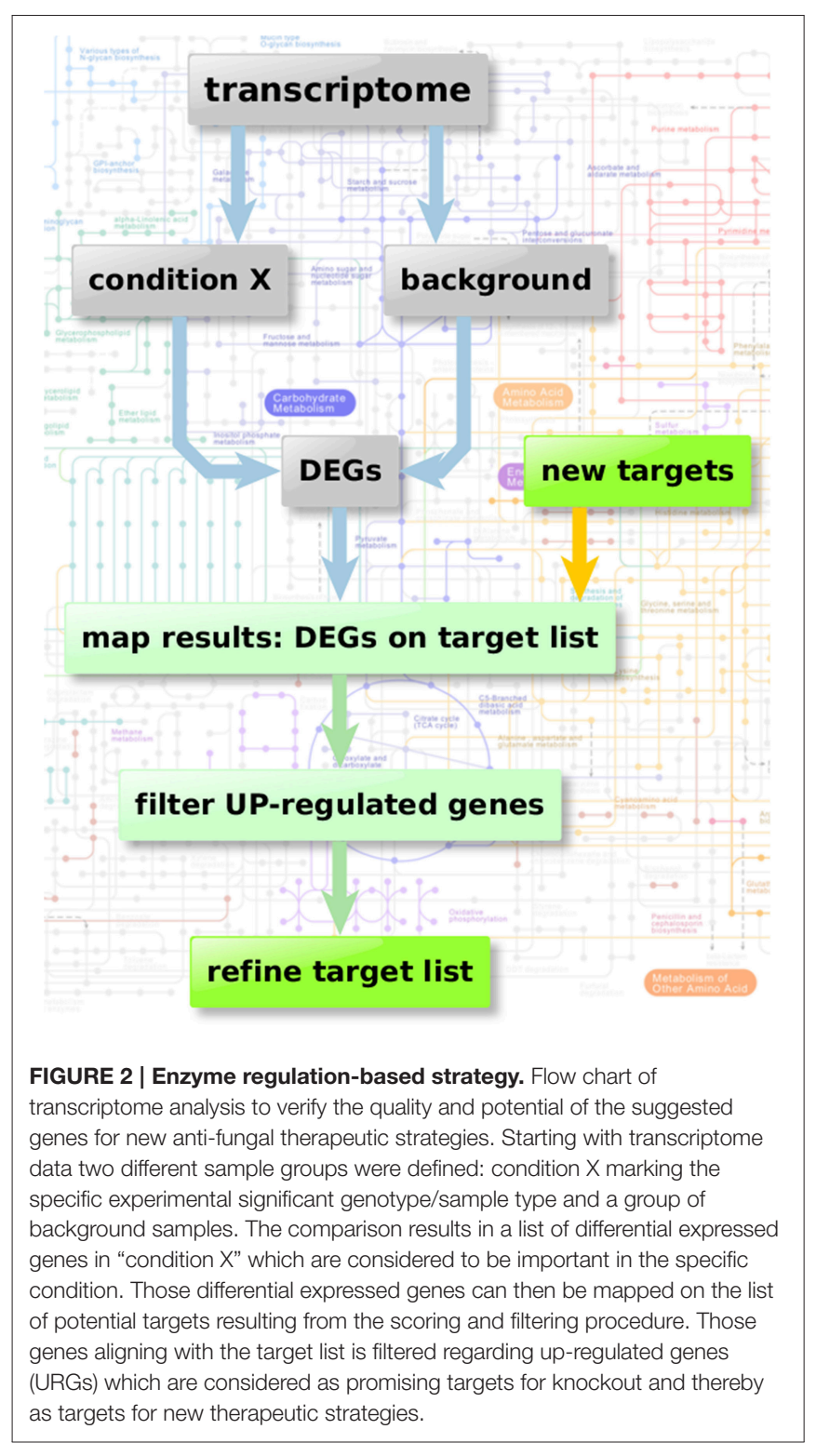

2008), by iron deficiency (Schrettl et al., 2010) and under hypoxia adaptation (Willger et al., 2008).

We next compared the expression level of all genes. Using GEO2R we calculated logarithmic fold change ( $\log \mathrm{FC})$-values as well as $p$-values for every gene. We modified the GEO2R output in the manner that the output only consisted of genes with a $p<0.05$. Those genes were considered as significantly differential expressed genes under given conditions.

In the next step, the resulting list of differential expressed genes was matched onto the list of potential targets (all $A$. fumigatus metabolic enzymes; later enriched subsets) to receive the relative expression of those target genes in comparison to control conditions.

The control condition was here in all comparisons the wild type of A. fumigatus grown in rich medium with wild type strains used as control being: AF293 for the data set analyzed from
Schrettl et al. (2010) and McDonagh et al. (2008); CEA10 in Willger et al. (2008)).

Furthermore, we analyzed the resulting expression list of target genes with respect to increased transcript level since up-regulated genes (URGs) are considered important genes for A. fumigatus growth under this condition, thus indicating potential antimycotic targets. Additionally we compared those up-regulated target genes with the metabolic simulation data to specify the quality of each suggested target.

\section{Protein-Protein Interaction-Based Strategy}

Three criteria were considered here in three sub-pipelines for in silico screening (Figure 3, top): Metabolic network hub or rim protein, targetable metabolic, or regulatory domains which have no ortholog in the host as well as available structure from the protein databank and drugs targeting the structure.

\section{Sequence Comparisons}

Sequence comparisons used standard techniques such as basic local alignment sequence tool (Altschul et al., 1997). We used the following parameters: A stringent $e$-value threshold 1e-10, a bit-score of over $>500$, word_size 6 , scoring matrix Blosum62, gap existence cost 11, gap extension cost 1 for the blastp based similarity searches followed by manual inspection of functional domains and query coverage to avoid misinterpretation.

\section{Protein Interaction Data}

Protein interaction data were taken from the DIP database (Database of Interacting Proteins; Salwinski et al., 2004). The orthologous protein sequences from A. fumigatus were mapped on the available interaction data from DIP using the interolog approach. All the orthologous interactions were predicted with OrthoMCL (Li et al., 2003; Fischer et al., 2011). The resulting interactions in A. fumigatus were further investigated to check which of them are supported by domain-domain interactions (DDI) using the DDI containing databases DOMINE (Yellaboina et al., 2011), DIMA 3.0 (Luo et al., 2011), and IDDI (Kim et al., 2012). The subcellular localization was predicted using an improved KnowPred software (Lin et al., 2009), the latest UniLoc server and SwissProt localization annotations for these proteins (bioapp.iis.sinica.edu.tw/UniLoc/). The localization information was further used as confirmatory evidence for plausible interacting proteins. Only the domain that supported interactions in which the interacting partners shared a minimum of one similar localization was considered as true proteinprotein interaction (PPI) in our reconstructed A. fumigatus interactome. The protein interaction network was reconstructed using Cytoscape (Smoot et al., 2011). Network analysis predicted highly connected nodes (hubs) and metabolic bottlenecks which suggested topologically important proteins which subsequently can be used as potential drug target if they do not have homology with human proteins. Of the top $20 \%$ proteins based on degree rank and betweenness rank were retrieved and re-ranked based on the minimum of their cumulative rank in the common top $20 \%$ proteins $S$

$$
\mathrm{X}_{\mathrm{r}}=\sum \mathrm{X}_{\mathrm{de}}+\mathrm{X}_{\mathrm{be}}
$$




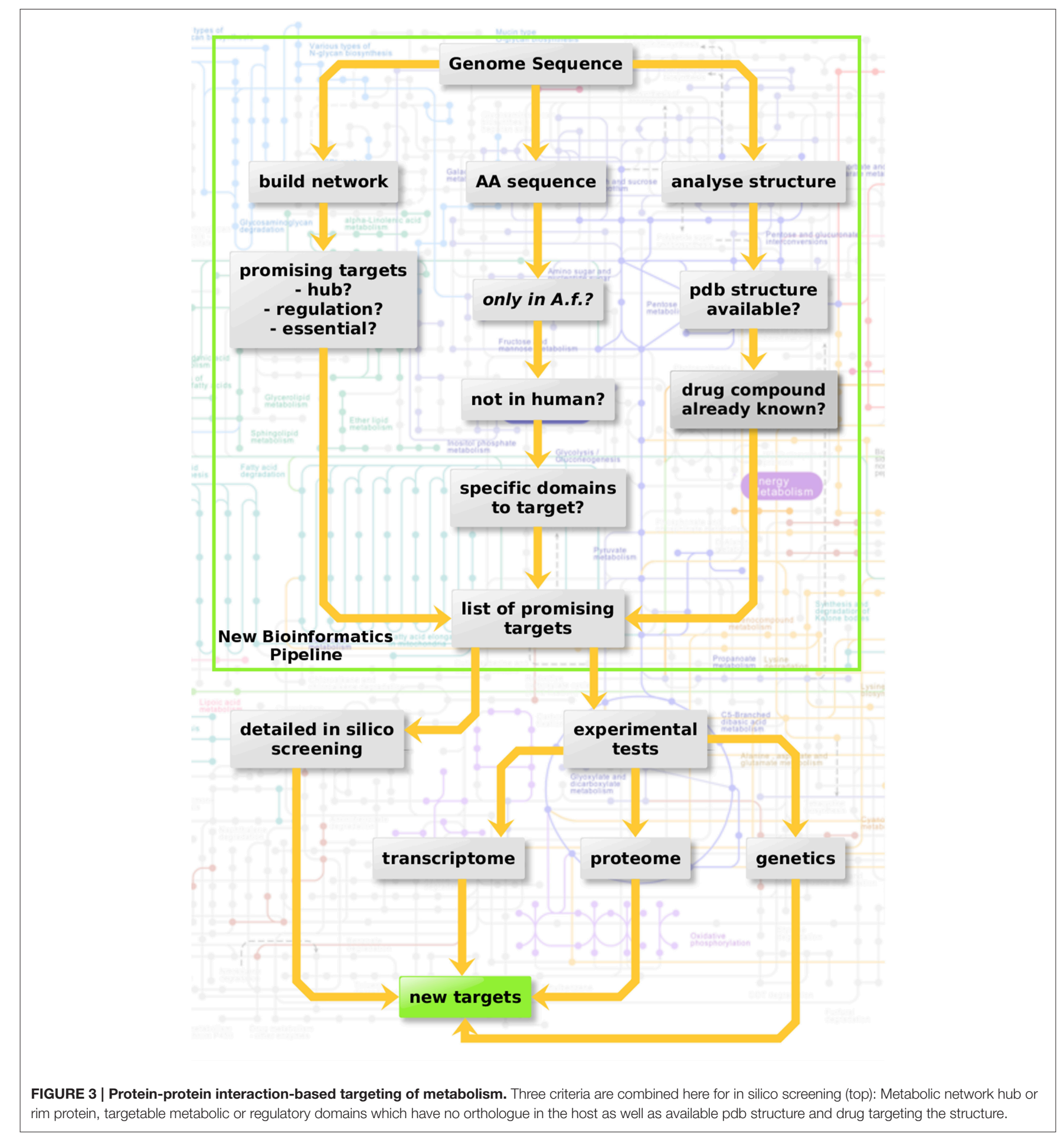

Where $X_{r}$ is the new rank of protein $X$, the index "de" refers to the degree rank of protein $\mathrm{X}$ and the index "be" refers to the betweenness rank of protein $\mathrm{X}$.

Metabolic bottlenecks are pathways and reactions for which no alternative routes exist, so all metabolic flux for the following metabolites has to go through such a bottleneck; these points are also called "choke points" (Rahman and Schomburg, 2006) and are a valid and promising position to interfere with primary metabolism.

Betweenness centrality looks how central a given protein ("vertex" or "node") is in a network. It counts the number of shortest paths from all vertices to all others that pass through that node. The degree rank just counts how well connected a protein target is by counting all edges leading to one of the vertex (so how 
many interactions converge on this protein). Further details are found in Thadakamalla et al. (2005).

The newly ranked protein were further compared in their sequence against the human proteome and the proteins showing significant similarity with human were discarded from the potential drug target list. The KEGG (Kanehisa and Goto, 2000; Kanehisa et al., 2014) database was used to annotate the proteins in the target list that have involvement in fungal metabolism.

\section{Drug Targets with Orthology to Functionally Important Genes}

Furthermore, the A. fumigatus proteome and eukaryotic proteins available at Database of Essential Genes (DEG) (Luo et al., 2013) were analyzed to identify orthologous protein using OrthoMCL (Li et al., 2003; Fischer et al., 2011). We further applied a BlastP (Altschul et al., 1997) based screening to filter out A. fumigatus protein in the set of orthology-based predicted important proteins that are significantly similar with human proteins. Next, the interacting as well as predicted essential proteins were metabolically annotated to establish the metabolic importance and metabolic pathway involvement. The final list of potential drug target were scored and ranked accordingly to the RhumPdb score (Toomey et al., 2009).

$$
R_{\text {humDB }}=\log _{10}\left(\frac{E_{\text {BlastP }}[\text { query vs human proteome }]}{E_{\text {Blastp }}[\text { query vs } P D B]}\right)
$$

A high $R_{\text {humPDB }}$ score indicates that the target has minimum similarity with human and has a close protein data bank $(P D B)$ structure template.

To identify and prioritize targets in the metabolism of A. fumigatus, first all metabolic reactions were mapped using the orthology information from the already available metabolic models of Aspergillus oryzae, Aspergillus niger, and Aspergillus nidulans (models by Andersen et al., 2008; David et al., 2008; Vongsangnak et al., 2008). Furthermore, reactions that were not annotated from the orthology were fetched using the Blast2GO (Conesa and Götz, 2008) annotation and Enzyme database (Bairoch, 2000). At every step manual verification was performed to remove any redundant information. The preliminary list of metabolic reactions was reduced based on the following criteria: (i) all those reactions which were catalyzed by true orthologs of human proteins were removed; (ii) the list was compared to the DEG database (Luo et al., 2013) of orthology based genes to ensure that no gene potentially vital for growth was removed from the list during the reduction process; (iii) for the enzymes also present in the interactome, the number of protein interactions these proteins have ("degree") was also considered during reduction as an enzyme with high degree (interacting with many proteins, connected to many pathways) has more possibility to be involved in multiple pathways and vice versa; (iv) as a key simplification, and to avoid combinatorial explosion during elementary mode calculation, we selected very few enzymes for any linear stretch while considering the metabolites which are similar; (v) the pace-maker (flux value determining) enzymes for long linear pathways were generally included; (vi) finally, pathway annotation was done for this list and only the reactions which participate in primary metabolic pathway were further considered to find the drug targets in primary metabolism. Moreover, we calculated the RhumPDB score (Toomey et al., 2009) and mapped the gene expression data (Bertuzzi et al., 2014) over the preliminary target list to prioritize the drug targets.

The following criteria were used to prioritize targets further: (i) genes that were highly expressed at many independent time points were given top priority (mean significant differential expression) over the genes highly expressed at fewer time points. A top expression rank represents a higher expression of a gene at all time points during invasive infection (ii) the final priority order was decided based on the minimum of RhumPDB (Toomey et al., 2009) and expression rank (iii) it was also noted whether the enzyme participates in a fungal-unique pathway or any pathway is shared with human metabolism. Proteins were ignored for which co-ortholog proteins were available, as this implies that the same reaction might be catalyzed by alternative proteins.

\section{RESULTS AND DISCUSSION}

\section{Targets from Metabolic Modeling to Interfere with Pathogen Proliferation}

We defined here that the targets which are critical in a metabolic sense to be easily identified by pointing out strong active pathways pertaining to different environmental conditions. Calculating the resulting differential effects on metabolite synthesis and growth then allows the identification of enzymes which are essential for growth and valid drug targets. To achieve a compact network regarding identification of new antimycotic targets in A. fumigatus, first we removed many non-central A. fumigatus metabolic reactions in vitamins, lipids, and amino acid metabolism that are governed by human orthologous proteins. The central carbohydrate metabolism consisting of glycolysis, PPP and TCA, however, was retained, as otherwise no flux calculations covering a major part of central metabolism are possible. We then used only those reactions for which we had availability of gene expression data during infection. Overall, the enzymes we selected in the analysis were based on their importance in metabolic pathways, pathway crosstalk and flux activity analysis in the model. With these criteria for filtering the resulting list consists of 162 unique metabolic reactions which were specific for A. fumigatus and that are catalyzed by 102 enzymes and contained 204 metabolites.

The resulting model of central primary metabolism for A. fumigatus is illustrated in Figure 4, and given in full detail in the Supplementary Material (input files for calculations with Metatool see Supplementary Table 1; all enzyme reactions considered are also given (Supplementary Table 15, with the mapping to gene identifiers given in Supplementary Table 19) so that from the stoichiometric matrix [Supplementary Table 16 or Supplementary Table 18 (null space version)] all elementary modes (Supplementary Table 17) can be calculated).

The model contains the central carbohydrate and amino acid metabolism, lipid synthesis and degradation as well as key reactions for intermediary metabolism and all necessary 


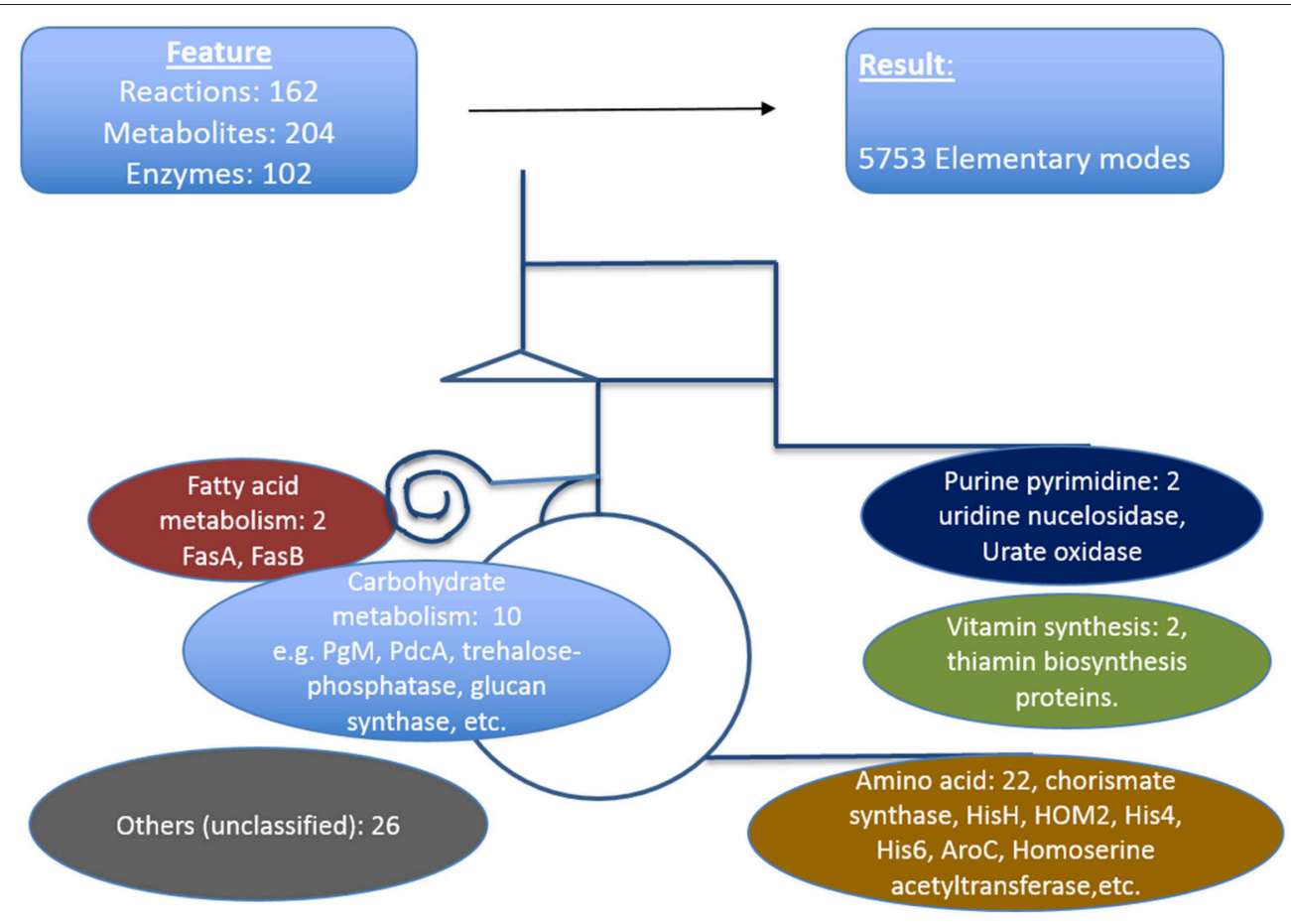

FIGURE 4 | Metabolic model of $\boldsymbol{A}$. fumigatus. This figure illustrates the metabolic web considered, focusing on primary metabolism. Top: Shown are reactions, metabolites and enzymes (left) and modes calculated (right). Below major pathways modeled are given together with the number and examples of identified promising antibiotic target structures. Details are found in Supplementary Material File 19 together with input files, stoichiometric matrix, calculated elementary modes, and flux values for optimal growth and biofilm condition.

cofactors, often vitamins. From a systems biology point of view there are hub metabolites (such as currency metabolites including ATP, but also pyruvate, glutamic acid), central enzyme nodes (for instance adenylate kinase to balance energy), unique pathways and reactions, which are similar as "choke points" (Rahman and Schomburg, 2006) to represent good positions for interfering with metabolism. Overall, our network involves 204 metabolites, 102 enzymes, and 5737 elementary modes (model in YANA/SBML format: Supplementary Table 20). Such a model serves as a basis for both targeting genes encoding metabolic enzymes as well as phenotyping with respect to growth or nutritional characteristics. Phenotyping including prediction of essential genes for growth requires a large model where most metabolic reactions in these pathways and their branching are considered so that there is good correlation between prediction and any observed phenotype. Furthermore, larger models allow identification of well-connected enzymes socalled hub enzymes. Further methods such as metabolic control theory allows to identify enzymes with a high metabolic control coefficient as well as refined modeling of flux changes in general.

Specific fluxes and changes, were calculated using YANA: As starting values for the flux calculation the flux value for each elementary mode was set to 1 ; gene expression data sets were next used to calculate affected key modes: The different expression values for each enzyme were used to coarsely approximate their different activity levels. Systematically different flux values for each elementary mode were combined using a genetic algorithm
(Schwarz et al., 2005) and steepest descent methods (Cecil et al., 2011) to minimize the error of the squared differences between calculated and approximated enzyme activity. Integration of gene expression data provides an acceptable way to calculate fluxes for those parts of the metabolic network which cannot be validated by directly measured metabolic data. We know that the average error for the flux value calculated from gene expression data is around $5-10 \%$ for the network, if a large scale data set is available and used on the pathways of interest and the simulation converges. This was validated by looking at and measuring differences in metabolite concentrations for the calculated flux values in several of our studies in infection biology (Cecil et al., 2011, 2015).

Recent studies have shown that the fungus A. fumigatus in its aerial grown biofilm-like state exhibits reduced susceptibility to antifungal drugs and undergoes major metabolic changes that are thought to be associated with virulence. These differences in pathological and physiological characteristics between biofilm and liquid shake conditions strengthen the notion that the planktonic state condition is a poor in vitro disease model. We therefore also used actual biofilm gene expression data from Muszkieta et al. (2013) for comparative calculations to the planktonic state datasets. Calculations were performed with YANAvergence as detailed in Materials and Methods to provide the resulting planktonic state fluxes (Supplementary File 2) and the gene expression under biofilm conditions (Supplementary File 4). That convergence was achieved and the data of the 
predicted fluxes correlate well with the gene expression data is shown in Supplementary File 3 (for the planktonic state) and Supplementary File 5 (for biofilm formation). Note that this calculation also reveals which enzymes in A. fumigatus are growth condition-specific higher or lower active (regarding their fluxes) as predicted just according to the gene expression data (all the points which are above the diagonal show higher gene expression ("experiment") then their calculated flux; see Supplementary Files 3, 5). Points below the diagonal indicate stronger flux then expected from the experimental data on gene expression. The detailed datasets are provided in Supplementary File 2 (planktonic growth, left: enzyme name, mapping to gene identifiers is given in Supplementary Table 19; middle: flux values compared to gene expression, right and the resulting regulatory difference, very right) and Supplementary File 4 (biofilm growth).

\section{Target Ranking and Identification}

According to the metabolic modeling we performed, pathways differentially regulated correlating with the biofilm gene expression datasets include: glycolysis (down-regulated) and gluconeogenesis (up-regulated, see for example: enolase flux is in the correct direction); down-regulatedlipid, fatty acid, and isoprenoid metabolism up-regulated, in particular ergosterol and cell wall synthesis, pentose phosphate cycle, nucleotide metabolism (signaling molecules), amino acid metabolism up-regulated and vitamin and cofactor metabolism downregulated, several degradation pathways upregulated (Table 1). Such strongly induced genes qualify (see M\&M; prioritization of targets) as potentially interesting targets for antimycotics as they are essential for growth, emerging from this approach as these enzymes carry a strong flux under biofilm formation (in that sense these are named "pace maker enzymes" as they determine the flux value and how strong this pathway is active). The Table 1 only provides examples for the top enzymes identified by this criterion. For optimal results, however, all criteria have to be compared and combined (see Results, part 4). In particular, we also considered from this approach the results from the elementary mode analysis and identified enzymes essential for growth by providing building blocks of primary metabolism required for growth.

\section{Targets from an Enzyme Regulation-Based Strategy}

We next show the flow chart for the enzyme regulation-based strategy (Figure 2). First, we use available transcriptome data to verify the quality and potential of the suggested genes and their resulting proteins as antifungal targets. To analyze transcriptome data two different conditions are usually compared: A specific expression condition $\mathrm{X}$ marking the specific experimental significant genotype/sample type and for comparison the control or background samples. The comparison results in a list of differential expressed genes. The user can apply different filters to identify promising targets shared or exclusive for one condition, up- or down regulation and of course the "no orthologs in man" criterion (to avoid side effects in man). Genes which are robustly expressed under many different conditions (true housekeeping genes) represent another possible targeting strategy.

Differential expressed genes can then be placed on the list of potential targets resulting from the scoring and filtering procedure. Those genes aligning with the target list are filtered regarding URGs which are considered as promising targets. The screen for different genes upregulated in the transcriptome for conditions such as iron deficiency (details in Supplementary File 8; Schrettl et al., 2010), hypoxia adaptation (see Supplementary File 7; Willger et al., 2008) and invasion initiation conditions (Supplementary File 6; McDonagh et al., 2008) shows different prominent metabolic genes significantly $(p<0.05$, multiple testing condition) up- (red) or down-regulated (green) under these condition (Table 2), the control is always wild type. This allows for the identification of genes which are crucial for survival (highly expressed) under those challenging conditions, as an antimycotic will be a similar stress. By targeting the major players involved in metabolic adaptation, we increase the likelihood of a strong inhibitory effect that may stop metabolism, inhibiting all growth and eventually killing the cell. This transcriptomebased target identification has also promise for a quite different strategy, targeting those genes which are critical for maximum growth under optimal conditions (green entries in Table 2).

\section{Target Ranking and Identification}

The following top enzyme targets were predicted from this approach, zooming in on lipid metabolism and amino acid

TABLE 1 | Top Targets from metabolic modeling to target pathogen metabolisma.

\begin{tabular}{|c|c|c|c|}
\hline Pathway & & Target by pace maker enzyme & Validated by flux analysis \\
\hline Vitamin & Riboflavin & RIB1 & Yes \\
\hline Nucleotide & Pyrimidin & Carbamoyl synthetase & Yes \\
\hline \multirow[t]{2}{*}{ Lipid } & Ergosterol and & Ergosterol synthetase & Yes \\
\hline & Cell wall synthesis & & Yes \\
\hline \multirow[t]{2}{*}{ Carbohydrates } & Glycolysis & Aldolase, enolase & Yes \\
\hline & Pentose Phosphate Cylce & Transketolase & Yes \\
\hline
\end{tabular}

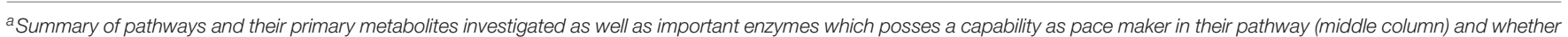

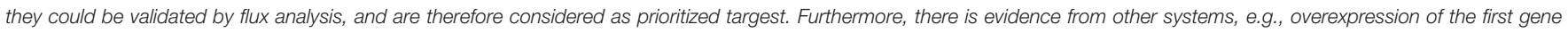

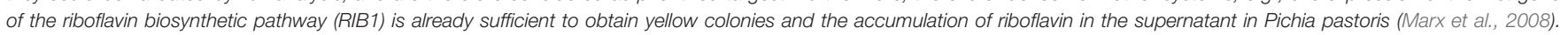

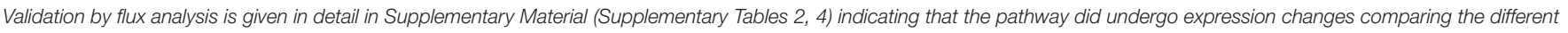
data sets against each other in the flux calculations. 
TABLE 2 | Metabolic gene expression under iron deficiency, invasion initiation, and hypoxia compared to controla .

\begin{tabular}{|c|c|c|c|c|c|}
\hline & Iron deficiency & In vivo & Hypoxia adaptation & Pathway & Annotation \\
\hline \multicolumn{6}{|l|}{ Sup6 } \\
\hline AFUA_3G04210 & 1.052 & -1.058 & -1.474 & FA & Fatty acid synthase alpha subunit FasA \\
\hline AFUA_3G04220 & 1.153 & -1.971 & n.s. & FA & Fatty acid synthase beta subunit \\
\hline AFUA_3G09290 & -0.706 & -0.719 & 1.479 & $\mathrm{AA}$ & Phosphoglycerate mutase, 2,3-bisphosphoglycerate-independent \\
\hline AFUA_3G06830 & n.s. & -1.684 & 0.945 & AA & Aspartate-semialdehyde dehydrogenase \\
\hline AFUA_1G14570 & n.s. & 1.042 & -2.718 & AA & Phosphoribosyl-AMP cyclohydrolase \\
\hline AFUA_4G06460 & n.s. & 2.834 & n.s. & $\mathrm{AA}$ & Pentafunctional AROM polypeptide \\
\hline AFUA_3G14490 & n.s. & -1.401 & -1.574 & $\mathrm{AA}$ & Ketol-acid reductoisomerase \\
\hline AFUA_5G05590 & n.s. & -1.060 & 1.703 & $\mathrm{AA}$ & Aspartokinase \\
\hline AFUA_5G05820 & n.s. & -2.065 & 1.107 & AA & Homoserine kinase \\
\hline AFUA_6G12400 & n.s. & -0.031 & -1.568 & SUC & 1,3-beta-glucan synthase catalytic subunit FksP \\
\hline \multicolumn{6}{|l|}{ Sup2 } \\
\hline AFUA_6G11390 & -0.926 & 0.459 & -2.196 & & 1,3-beta-glucanosyltransferase Gel2 \\
\hline AFUA_3G11070 & 0.987 & -4.248 & n.s. & GLU & Pyruvate decarboxylase PdcA \\
\hline AFUA_2G10740 & n.s. & -1.164 & n.s. & VIT & Thiamin biosynthesis protein (Thi-4) \\
\hline AFUA_5G12190 & n.s. & -4.310 & -0.817 & & Transcription initiation factor subunit (TAF30) \\
\hline AFUA_4G09660 & n.s. & 1.849 & n.s. & & Secretory component protein shr3 \\
\hline AFUA_6G12400 & n.s. & -0.031 & -1.568 & SUC & 1,3-beta-glucan synthase catalytic subunit FksP \\
\hline AFUA_1G06700 & n.s. & -1.705 & n.s. & & Metacaspase CasA \\
\hline AFUA_3G14140 & 0.367 & -1.273 & -1.262 & & Metacaspase CasB \\
\hline AFUA_4G13340 & n.s. & -0.008 & -1.412 & & DUF907 domain protein \\
\hline AFUA_2G17650 & -0.523 & 2.921 & -2.795 & & DUF907 domain protein \\
\hline AFUA_2G17300 & n.s. & 4.050 & 1.057 & GSH & Glutathione S-transferase \\
\hline AFUA_2G09040 & n.s. & 1.360 & 1.053 & & Vacuolar transporter chaperone (Vtc4) \\
\hline AFUA_2G04010 & n.s. & -1.125 & 1.182 & SUC & Alpha,alpha-trehalose-phosphate synthase subunit \\
\hline \multicolumn{6}{|c|}{ ESSENTIAL GENES } \\
\hline AFUA_3G14440 & 0.916 & -1.174 & n.s. & & Cytochrome c oxidase family protein \\
\hline AFUA_4G10480 & n.s. & -2.375 & 1.812 & & Mitochondrial large ribosomal subunit protein L30 \\
\hline logFC: -1 & & O/NA & & 1 & \\
\hline
\end{tabular}

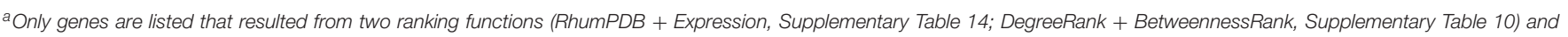

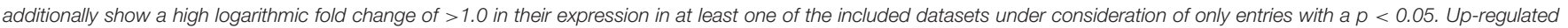

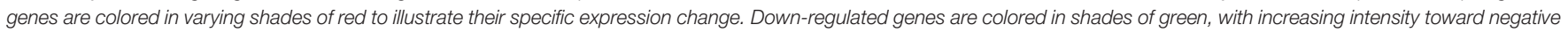

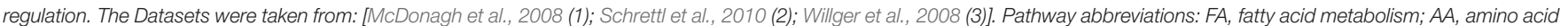
metabolism; SUC, sugar modifications; GSH, glutathione metabolism; GLU, gluconeogenesis.

metabolism and considering only significantly differentially expressed genes $(p<0.05)$ and only strongly regulated genes $(\log \mathrm{FC}>1)$ and ranking all genes and potential targets accordingly.

Fatty acid synthases are down-regulated in vivo and under hypoxic conditions but up-regulated under iron deficiency conditions. In contrast, phosphoglycerate mutase and aspartokinase, and homoserine kinase (amino acid metabolism) are down-regulated in vivo, while they are up-regulated under hypoxia adaptation. Furthermore, in vitamin metabolism, thiamin biosynthesis proteins stand out, showing a negative regulation under in vivo condition (similar to the biofilm condition investigated in results, part 1). Finally, several cell wall carbohydrate metabolism genes stand out as strongly regulated and induced both for invasion and under hypoxia, such as glutathione S-transferase and the vacuolar transporter chaperone (Vtc4).
In summary, in this pipeline we show only targets according to one simple but clear criterion: High expression under infectionassociated conditions as detailed above. However, other criteria help to identify alternative targets. As detailed in methods, we are currently screening for new targets with constant and high expression under several conditions, representing so-called housekeeping genes. As this involves analysis of much more transcriptome data sets, a definite list of metabolic target enzymes will be generated during future efforts.

\section{Targets from Protein-Protein Interaction-Based Targeting of Metabolism}

As a third line of research we considered an interactome-based approach. Figure 5 shows the protein-protein interaction-based drug targets.

The final interactome of $A$. fumigatus after considering the domain-domain interaction database (DDI) and localization 


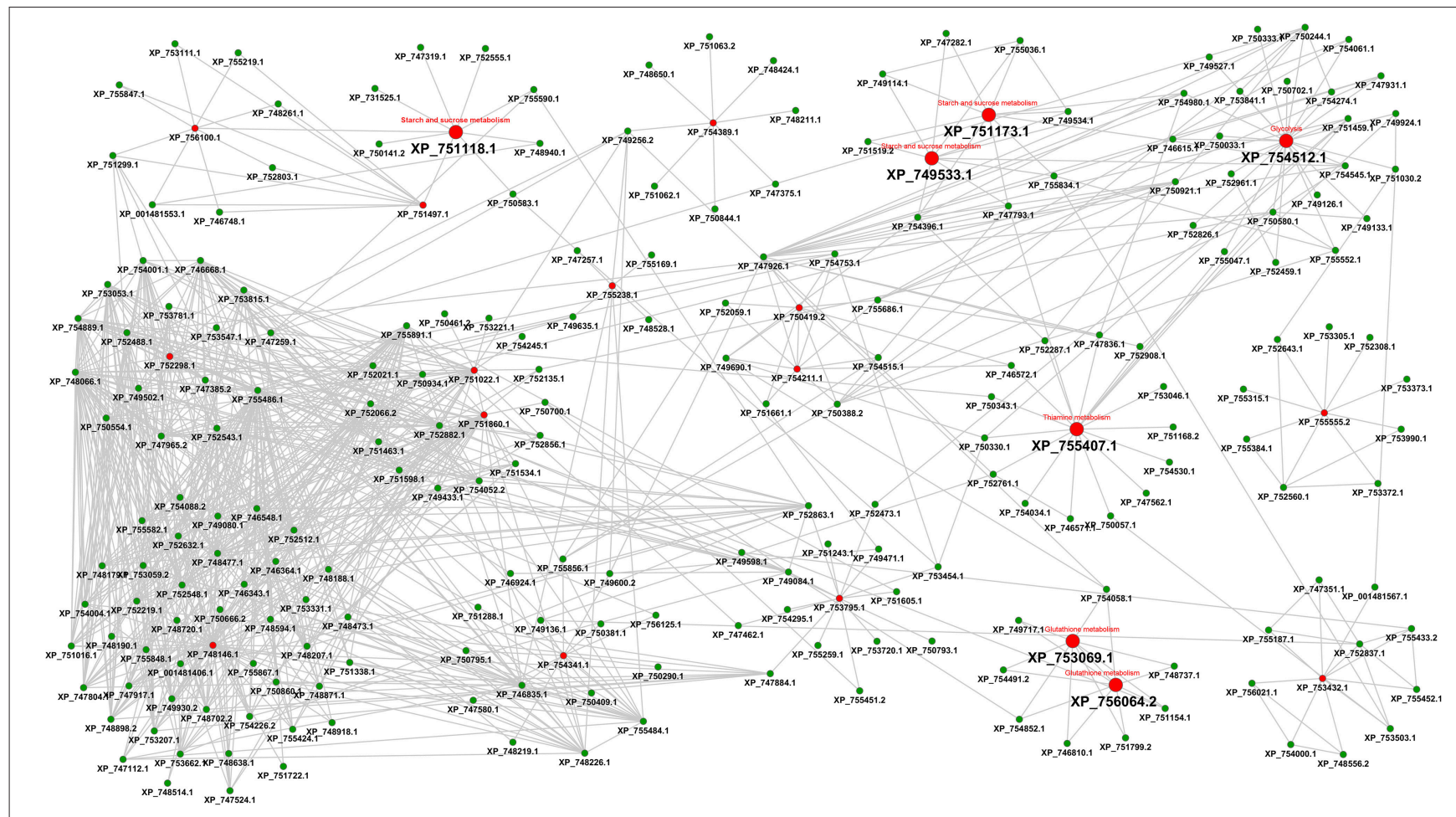

FIGURE 5 | Interactome view of metabolic proteins for antifungal targeting. Red color nodes depict the network based drug targets in A. fumigatus interactome. The targets associated with the metabolism are shown with big red nodes with associated metabolic pathway annotation.

filtering consists of 1903 nodes and 4743 PPIs and its interactome relation is given in Supplementary Table 9 (the interactome can also be analyzed in detail for hub proteins, bystanders, diameter, GO-categories etc., hence this data is made available here for such different research questions). The topologically important top $20 \%$ proteins were selected based on cumulative rank of hub degree rank and betweenness rank. Among these, 21 proteins were found to lack any significant similarity with human proteins, and are therefore proposed as preliminary network based drug targets (Table 3A). A subnetwork consisting of the proposed network based drug targets is shown in Figure 5. Out of 21 targets seven were annotated to be involved in metabolic pathways.

After consideration of the added Ranking scores from Degree Rank and Betweenness Rank and sorting of the resulting list we re-ranked the genes regarding their position. The five best hits include (1) 1,3-beta-glucosyltranferase Gel2 (AFUA_6G11390), (2) phosphate transporter (Pho88, AFUA_5G01960), (3) DUF6 domain protein (AFUA_1G09310), (4) pyruvate decarboxylase PdcA (AFUA_3G11070) as well as (5) high osmolarity signaling protein Sho1 (AFUA_5G08420).

In the subsequent analysis we determined a further ranking based on RhumPDB data and expression by calculation of the RhumPDB Score (Table 3B). The combination of those two values by adding them up lead us to a new rank. We then assigned them a priority order related to their position in the list. The five top hits resulting from this method are (1) chorismate synthase (AFUA_1G06940), (2) fatty acid synthase alpha subunit FasA (AFUA_3G04210), (3) urate oxydase UaZ (AFUA_2G10520), (4) glutamine amidotransferase:cyclase (AFUA_2G06230), and (5) fatty acid synthase beta subunit (AFUA_3G04220).

Hence, a total of 65 proteins were predicted to be important based on orthology with DEG(“database of essential genes")derived proteins.

Genes in the DEG database were included if they had scored to be essential for one organism under a specific condition. In general, essentiality depends very much on the environmental condition tested. Instead, the sequence similarity to the DEG entry can be determined by objective criteria. It is thus hard to predict essentiality of the A. fumigatus homolog. For the A. fumigatus genes we hence used DEG only to identify functionally conserved and probably important metabolic genes. Two preliminary targets identified in this way include the 50S ribosomal protein (AFUA_4G10480) and a cytochrome c oxidase family protein (AFUA_3G14440) that participates in oxidative phosphorylation.

\section{Target Ranking and Identification}

A total of 130 metabolism-associated A. fumigatus proteins were identified and subsequently parsed and prioritized regarding contained top targets (see Materials and Methods). The gene expression of predicted potential targets in the infection condition (Bertuzzi et al., 2014) was used to rank the genes 
TABLE 3 | Results from two different ranking functions of the interactome pipeline ${ }^{a}$.

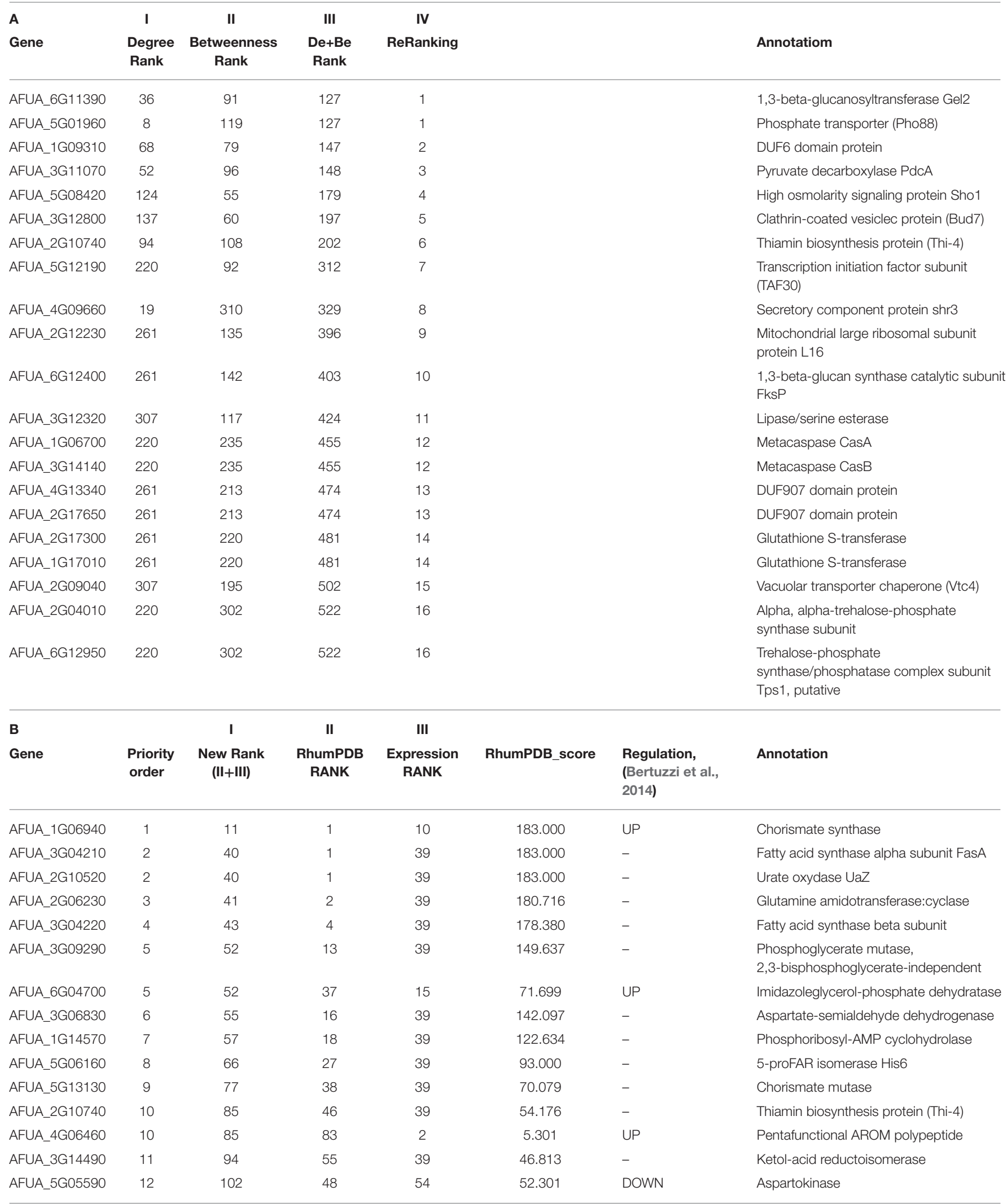


TABLE 3 | Continued

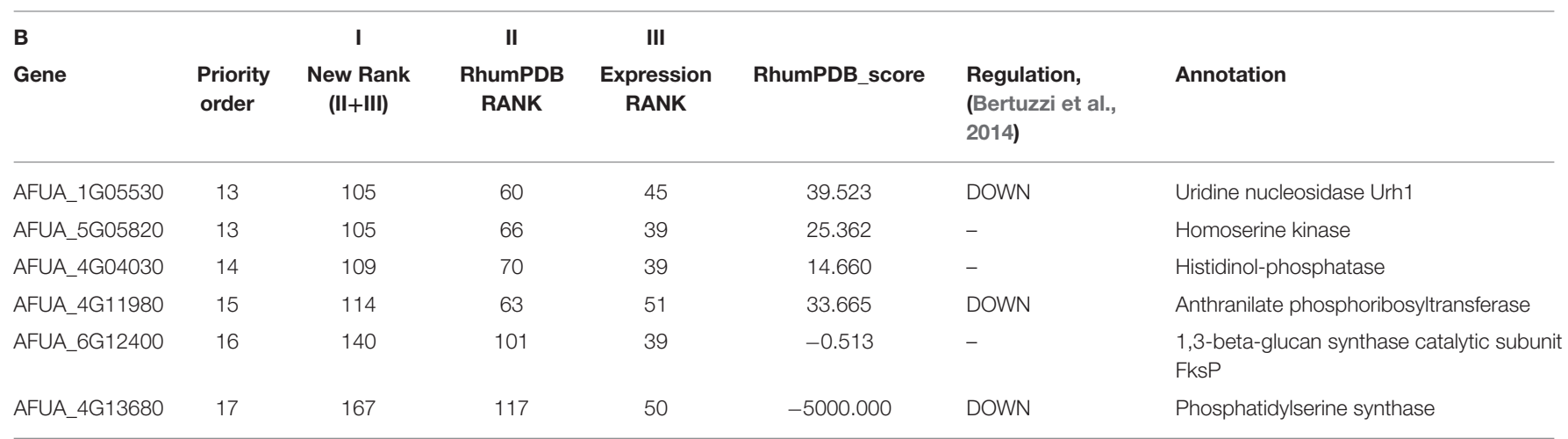

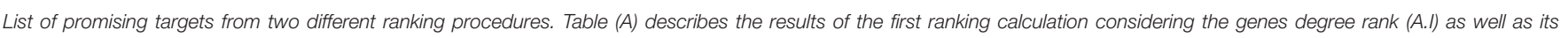

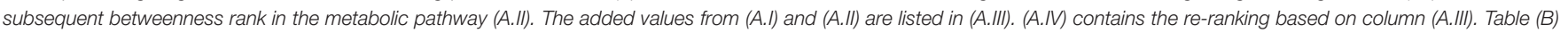

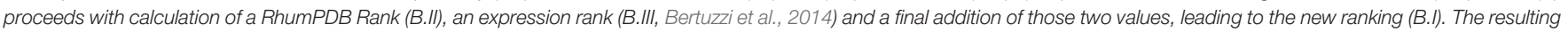

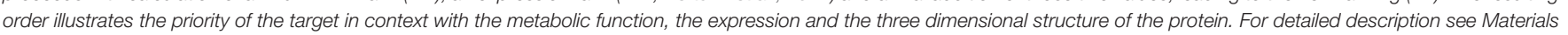
and Methods.

according to their importance in infection and metabolism. The genes that were highly expressed at all four time point were given top priority (mean significant differential expression) then the gene highly expressed at three time points were ranked and so further on. A top expression rank represents a high gene expression at all time points during invasive infection (Supplementary Table 11). We also analyzed the RhumPDB score for targets and together with expression rank this score was used for prioritization. The score represents the normalized $\log 10$ ratio of BlastP $e$-value of $A$. fumigatus vs. human proteome and A. fumigatus vs. current PDB structures (Supplementary Table 12). The RhumPDB score orders the targets based on their higher closeness to crystallized PDB structures and their lack of similarity to human proteins. As a final reduction of the targets we implemented sequence similarity searches of A. fumigatus against human proteins by blast to avoid any off-targets. The genes/enzymes catalyzing the reactions that can also be catalyzed by alternative enzymes are indicated. Therefore, we also ignored such enzymes from our target list. The RhumPDB score and expression rank of the analyzed 130 metabolic proteins is listed in Supplementary Table 13. The final list consists of 22 proposed targets from fungal metabolic pathways (Supplementary Table 14).

As an example for a target list entry, PdcA (Pyruvate decarboxylase, AFUA_3G11070), participates in biofilm formation, which is represented by data from Muszkieta et al. (2013). PdcA is down-regulated three fold during biofilm formation. Hence, in principle, this target could also be picked up by a transcriptomics approach, but only if a different filter from our example is used, for instance strongly regulated genes, comparing wild type and biofilm formation. Other genes in our current list are not differentially expressed according to the Muszkieta dataset, so they are complementary found to those targets from the transcriptomic approach if using the filters just given.

The RhumPDB rank of this gene is 55, however, the protein is downregulated during invasive aspergillosis conditions and upregulated under iron limiting conditions, henceforth it might be possibly represent a valid target. AFUA_3G04210 (FasA Fatty acid synthase A) and AFUA_3G04220 (FasB Fatty acid synthase beta subunit) with RhumPDB rank of 1 and 4, respectively, significant expression changes, up-regulation in iron limiting condition and downregulation during invasion might be further promising targets. AFUA_3G14440 (cytochrome c oxidase family protein) from DEG can be examined as supported by expression changes under both tested conditions. These proteins would be the most interesting ones from this refined interaction-based pipeline to identify targets in the pathogen metabolism network (Figure 5).

In summary, we considered only top ranked proteins from each of the three sub-pipelines using interactomic data considering whether the target was connected to metabolism: either using structure and RhumPDB score (top seven targets all metabolic), or using combined degree rank and betweenness rank (top five targets given) as well as functional important, non-orthologous genes in the interactome (top two targets included).

\section{Identified Top Targets using Expert Knowledge and Combined Evaluation of the Three Pipelines}

In general, however, detailed knowledge and extensive data support the target strategy, which is in fact essential for any new antifungal development. We show this now combining our three pipelines: metabolic pathway modeling and flux calculations, gene expression, and transcriptome data on metabolic adaptation as well as network modeling and protein interactions. All three focus on the following metabolic areas: (a) vitamin synthesis, (b) lipid synthesis, and (c) biosynthesis of amino acids. This focus was chosen as A. fumigatus differs in these pathways significantly from the host, which is of course a simplified search for metabolic targets. However, for a direct comparison of the three pipelines (and sub-pipelines) this focus is valid as it allows to combine and 
TABLE 4 | Top Targets from protein-protein interaction-based targeting of metabolism ${ }^{\mathrm{a}}$.

\begin{tabular}{|c|c|c|c|c|c|}
\hline & Iron deficiency & In vivo & Hypoxia adaptation & Pathway & Annotation \\
\hline \multicolumn{6}{|l|}{ Sup14 } \\
\hline AFUA_3G04210 & 1.052 & -1.058 & -1.474 & FA & Fatty acid synthase alpha subunit FasA \\
\hline AFUA_3G04220 & 1.153 & -1.971 & n.s. & FA & Fatty acid synthase beta subunit \\
\hline AFUA_3G09290 & -0.706 & -0.719 & 1.479 & AA & Phosphoglycerate mutase, 2,3-bisphosphoglycerate-independent \\
\hline AFUA_1G14570 & n.s. & 1.042 & -2.718 & AA & Phosphoribosyl-AMP cyclohydrolase \\
\hline AFUA_4G06460 & n.s. & 2.834 & n.s. & $\mathrm{AA}$ & Pentafunctional AROM polypeptide \\
\hline AFUA_5G05590 & n.s. & -1.060 & 1.703 & $\mathrm{AA}$ & Aspartokinase \\
\hline AFUA_5G05820 & n.s. & -2.065 & 1.107 & $\mathrm{AA}$ & Homoserine kinase \\
\hline \multicolumn{6}{|l|}{ Sup10 } \\
\hline AFUA_4G09660 & n.s. & 1.849 & n.s. & & Secretory component protein shr3 \\
\hline AFUA_2G17650 & -0.523 & 2.921 & -2.795 & & DUF907 domain protein \\
\hline AFUA_2G17300 & n.s. & 4.050 & 1.057 & GSH & Glutathione S-transferase \\
\hline AFUA_2G09040 & n.s. & 1.360 & 1.053 & SUC & Vacuolar transporter chaperone (Vtc4) \\
\hline AFUA_2G04010 & n.s. & -1.125 & 1.182 & & Alpha, alpha-trehalose-phosphate synthase subunit \\
\hline \multicolumn{6}{|c|}{ ESSENTIAL GENES } \\
\hline AFUA_3G14440 & 0.916 & -1.174 & n.s. & & Cytochrome c oxidase family protein \\
\hline AFUA_4G10480 & n.s. & -2.375 & 1.812 & & Mitochondrial large ribosomal subunit protein L30 \\
\hline logFC: -1 & & O/NA & & 1 & \\
\hline
\end{tabular}

${ }^{a}$ Results of comparison of orthology results and transcriptomic results in consideration of metabolic function. Only differentially expressed genes with a confidence $p<0.05$ are shown. Up-regulated genes are colored in varying shades of red to illustrate their specific expression change. The Datasets were taken from: [McDonagh et al., 2008 (1); Schrettl et al., 2010 (2); Willger et al., 2008 (3)]. Pathway abbreviations: FA, fatty acid metabolism; AA, amino acid metabolism; SUC, sugar modifications; GSH, glutathione metabolism; GLU, gluconeogenesis.

compare results from all three approaches pertaining to the same pathways (Table 4). The top seven genes where selected from the resulting list of the structure analysis methodology, calculating the RhumPDB score to assess the quality of the target suggestion. Using this calculation the most accurate results can be obtained (Table 4; Supplementary 14). All of the listed genes are involved in metabolic pathways.

Furthermore, we selected the top five of the resulting genes from network analysis strategy. Two of them are also known to participate in metabolic pathways. This methods accuracy is highly depending on the network structure to calculate valid targets but nevertheless could give two new metabolic targets (Table 4; Supplementary 10).

The remaining method of the pipeline is relevant for the assessment of essential genes with no human orthologs. The two best hits can be easily assigned to a metabolic pathway (Table 4; essential genes) and hence can be assumed as valid target candidates for antifungal therapy.

Using the results of the analysis for differentially expressed genes in three different datasets (McDonagh et al., 2008; Schrettl et al., 2010, accession GSE22052; Willger et al., 2008, accession GSE12376), we used a stringent cutoff of a $p$-value of 0.05 and a logarithmic fold change of $>1.0$ or respective $<-1.0$ (Table 4).

In a first approach we mapped the resulting genes from Supplementary Table 10 and Supplementary Table 14 onto the gene expression results from Supplementary Tables 6-8. In addition to the resulting list from Supplementary Tables 10, 14 we also included some genes which according to common knowledge (DEG, Luo et al., 2013) are considered as essential.
Table 5 contains thus all genes that show differential expression values as well as significant regulation $(\log \mathrm{FC}>1$; $\log \mathrm{FC}<-1)$.

Further targets which have no or only low orthology to human ones and additionally show high expression change in at least one condition (iron deficiency, in vivo/invasion initiation, hypoxia adaptation) are shown in the list. We also included two essential genes, AFUA_3G14440 and AFUA_4G10480. Additionally we considered enzymes which have metabolic pathway neighborhood to already known (according to literature and biochemical data) promising antimycotic targets, as targeting further members of such a known pathway promising to be targeted by an antimycotic may yield further attractive targets (Table 5).

\section{Target Ranking and Identification Considering All Approaches}

Table 5 only shows new targets remaining after selection in the pipeline and its major steps: (a) the metabolic pipeline, (b) the orthology analysis (Supplementary Tables 10, 14), and (c) the transcriptome dataset analysis $(\log \mathrm{FC}>/<1.0 /-1.0 ; p<0.05)$. The results of the metabolic model simulations are shown in an additional column, listing the pathway annotations of the proteins (as far as known) as well as their predicted activation (red: positive, green: negative). We show only the targets with a top rank which were still (after filtering as above) considered by our analysis to be worth testing experimentally. This list of 64 targets is thus not further ranked as in the interactome pipeline (ranking see above) but rather the top targets from all approaches are considered equally. 
TABLE 5 | Singled out best targets from the combined pipeline regarding A. fumigatus ${ }^{\mathrm{a}}$.

\begin{tabular}{|c|c|c|c|c|c|c|c|c|}
\hline Gene & $\begin{array}{l}\text { Iron } \\
\text { deficieny }\end{array}$ & In vivo & $\begin{array}{l}\text { Hypoxia } \\
\text { adapt. }\end{array}$ & $\begin{array}{l}\text { Metabolic } \\
\text { category }\end{array}$ & $\begin{array}{l}\text { Evidence in } \\
\text { literature }\end{array}$ & $\begin{array}{c}\text { Genetic } \\
\text { test } \\
\text { worthwhile }\end{array}$ & $\begin{array}{l}\text { Currently } \\
\text { tested }\end{array}$ & Annotation \\
\hline AFUA_1G06940 & 0.000 & 0.820 & -1.855 & AA & & & 19 & Chorismate synthase (ARO2) \\
\hline AFUA_3G04210 & 1.052 & -1.058 & -1.474 & FA & 1 & & & Fatty acid synthase alpha subunit FasA \\
\hline AFUA_2G10520 & 0.000 & -0.757 & -3.258 & CAF & 2.3 & & & Urate oxydase UaZ \\
\hline AFUA_3G04220 & 1.153 & -1.971 & 0.000 & FA & 1 & & & Fatty acid synthase beta subunit (FasB) \\
\hline AFUA_3G09290 & -0.706 & -0.719 & 1.479 & AA & & & & $\begin{array}{l}\text { Phosphoglycerate mutase, } \\
\text { 2,3-bisphosphoglycerate-independent }\end{array}$ \\
\hline AFUA_6G04700 & 0.000 & -1.013 & 1.162 & AA;GLU & & & & Imidazoleglycerol-phosphate dehydratase (HisB) \\
\hline AFUA_3G06830 & 0.000 & -1.684 & 0.945 & AA;GLU & & & & Aspartate-semialdehyde dehydrogenase (HOM2) \\
\hline AFUA_4G06460 & 0.000 & 2.834 & 0.000 & AA_a & & & & Pentafunctional AROM polypeptide (ARO1) \\
\hline AFUA_3G14490 & 0.000 & -1.401 & -1.574 & AA & & & & Ketol-acid reductoisomerase (ILV5) \\
\hline AFUA_5G05590 & 0.000 & -1.060 & 1.703 & AA_S & & & & Aspartokinase (HOM3) \\
\hline AFUA_1G05530 & 0.000 & 0.147 & 0.000 & PYR & & & & Uridine nucleosidase Urh1 \\
\hline AFUA_5G05820 & 0.000 & -2.065 & 1.107 & AA_s & & & & Homoserine kinase (THR1) \\
\hline AFUA_4G04030 & 0.000 & NA & 0.000 & AA_HIS & & & & Histidinol-phosphatase (HIS2) \\
\hline AFUA_4G11980 & 0.000 & 0.083 & 0.000 & AA_a & & & & Anthranilate phosphoribosyltransferase (TRP4) \\
\hline *AFUA_6G12400 & 0.000 & -0.031 & -1.568 & GLU & 6 & & & 1,3-beta-glucan synthase catalytic subunit FksP \\
\hline AFUA_4G13680 & 0.000 & -0.112 & 0.000 & AA_S & & & & Phosphatidylserine synthase \\
\hline \multicolumn{9}{|l|}{ Sup2 } \\
\hline AFUA_5G12190 & 0.000 & -4.310 & -0.817 & & & & & Transcription initiation factor subunit (TAF30) \\
\hline AFUA_4G09660 & 0.000 & 1.849 & 0.000 & & & & & Secretory component protein shr3 \\
\hline AFUA_2G12230 & 0.000 & -0.415 & 0.924 & & & & & Mitochondrial large ribosomal subunit protein L16 \\
\hline *AFUA_6G12400 & 0.000 & -0.031 & -1.568 & SUC & 6 & & & 1,3-beta-glucan synthase catalytic subunit FksP \\
\hline AFUA_3G12320 & 0.000 & 0.506 & 0.837 & & & & & Lipase/serine esterase \\
\hline AFUA_1G06700 & 0.000 & -1.705 & 0.000 & & 10 & & & Metacaspase CasA \\
\hline AFUA_3G14140 & 0.367 & -1.273 & -1.262 & & 10 & & & Metacaspase CasB \\
\hline AFUA_4G13340 & 0.000 & -0.008 & -1.412 & & & & & DUF907 domain protein (FlcA) \\
\hline AFUA_2G17650 & -0.523 & 2.921 & -2.795 & & & & & DUF907 domain protein (FlcA) \\
\hline AFUA_2G17300 & 0.000 & 4.050 & 1.057 & GSH & & & & Glutathione S-transferase \\
\hline AFUA_1G17010 & 0.000 & 0.681 & 0.000 & GSH & & & & Glutathione S-transferase \\
\hline AFUA_2G09040 & 0.000 & 1.360 & 1.053 & & & & & vacuolar transporter chaperone (Vtc4) \\
\hline AFUA_2G04010 & 0.000 & -1.125 & 1.182 & SUC & 11 & & & $\begin{array}{l}\text { Alpha, alpha-trehalose-phosphate synthase } \\
\text { subunit (tpsA/B) }\end{array}$ \\
\hline AFUA_6G12950 & 0.327 & -0.773 & 0.000 & SUC & 11 & & & $\begin{array}{l}\text { Trehalose-phosphate synthase/phosphatase } \\
\text { complex subunit Tps1 }\end{array}$ \\
\hline
\end{tabular}


TABLE 5 | Continued

\begin{tabular}{cccccccc}
\hline Gene & $\begin{array}{c}\text { Iron } \\
\text { deficieny }\end{array}$ & In vivo & $\begin{array}{c}\text { Hypoxia } \\
\text { adapt. }\end{array}$ & $\begin{array}{l}\text { Metabolic } \\
\text { category }\end{array}$ & $\begin{array}{l}\text { Evidence in } \\
\text { literature }\end{array}$ & $\begin{array}{c}\text { Genetic } \\
\text { test } \\
\text { worthwhile }\end{array}$ & $\begin{array}{c}\text { Currently Annotation } \\
\text { tested }\end{array}$
\end{tabular}

\begin{tabular}{|c|c|c|c|c|c|c|}
\hline \multicolumn{7}{|c|}{ ESSENTIAL GENES } \\
\hline AFUA_3G14440 & 0.916 & -1.174 & \multicolumn{3}{|l|}{0.000} & Cytochrome c oxidase family protein \\
\hline AFUA_4G10480 & 0.000 & -2.375 & \multicolumn{3}{|l|}{1.812} & Mitochondrial large ribosomal subunit protein L30 \\
\hline \multicolumn{7}{|c|}{ FURTHER CONSIDERED TARGETS } \\
\hline AFUA_2G15970 & 0.000 & -1.561 & 0.000 & \multicolumn{2}{|l|}{ PCS } & Phosphatidylethanolamine N-methyltransferase \\
\hline AFUA_5G05690 & 0.000 & 2.177 & 0.000 & \multicolumn{2}{|l|}{ AA } & Prephenate dehydratase \\
\hline AFUA_1G02110 & -0.832 & 0.086 & 0.000 & \multicolumn{2}{|l|}{ AA_a } & 3-deoxy-7-phosphoheptulonate synthase \\
\hline AFUA_7G04500 & 0.000 & 0.415 & 0.000 & \multicolumn{2}{|c|}{ AA_HIS } & ATP phosphoribosyltransferase \\
\hline AFUA_3G11640 & 0.000 & 0.914 & 0.986 & \multicolumn{2}{|c|}{$\mathrm{AA}$} & Homoserine dehydrogenase \\
\hline AFUA_4G10460 & 0.589 & -0.266 & 2.419 & \multicolumn{2}{|l|}{$\mathrm{AA}$} & Homocitrate synthase \\
\hline AFUA_5G07210 & 0.000 & -0.588 & 1.909 & \multicolumn{2}{|l|}{ AA } & Homoserine O-acetyltransferase \\
\hline AFUA_4G07360 & -0.622 & 0.052 & 0.000 & \multicolumn{2}{|l|}{$\mathrm{AA}$} & Methionine synthase \\
\hline AFUA_6G02860 & 0.000 & 1.827 & 1.566 & GLU & \multirow[t]{2}{*}{12} & 2-methylisocitrate lyase \\
\hline AFUA_6G03730 & -0.668 & 2.433 & 1.286 & $\mathrm{AA}$ & & 2-methylcitrate hydrolyase \\
\hline AFUA_1G09050 & 0.000 & -1.883 & 0.000 & \multirow[t]{10}{*}{ PCS } & 13 & Methylene-fatty-acyl-phospholipid synthase \\
\hline AFUA_4G12990 & 0.000 & 1.271 & 0.000 & & \multirow[t]{3}{*}{$14-16$} & Thioredoxin reductase \\
\hline AFUA_2G11290 & -0.452 & -0.573 & 0.000 & & & Orotate phosphoribosyltransferase 1 \\
\hline AFUA_4G12600 & 0.000 & -0.294 & 2.812 & & & Phosphoribosylaminoimidazole carboxylase \\
\hline AFUA_3G05650 & 0.000 & 0.474 & 1.158 & & \multirow[t]{5}{*}{17} & Trehalose-phosphatase \\
\hline AFUA_7G01220 & 0.000 & -1.010 & 1.083 & & & Squalene synthase \\
\hline AFUA_5G10680 & 0.000 & -0.203 & 0.000 & & & Phosphomevalonate kinase \\
\hline AFUA_3G05730 & 0.000 & 0.024 & 0.000 & & & Nicotinate mononucleotide pyrophosphorylase \\
\hline AFUA_5G08120 & -0.402 & 3.931 & 1.030 & & & Glutamate N-acetyltransferase \\
\hline AFUA_2G10660 & -1.133 & -0.890 & 0.000 & & \multirow[t]{2}{*}{18} & Mannitol-1-phosphate 5-dehydrogenas \\
\hline logFC: -3 & & O/NA & & \multicolumn{2}{|l|}{3} & \\
\hline
\end{tabular}

${ }^{*}$ Gene result in Sup10 + Sup14.

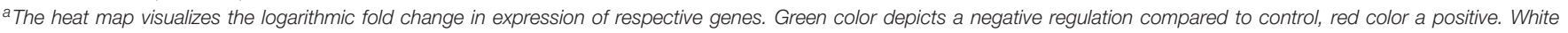

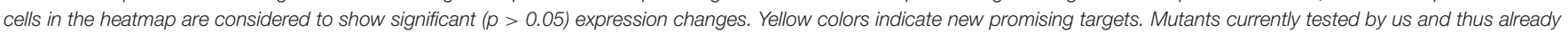

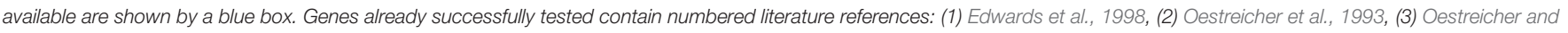

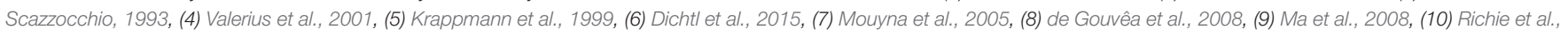

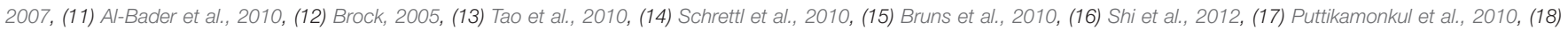

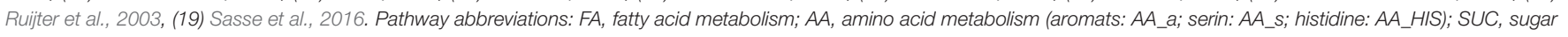
modifications; GSH, glutathione metabolism; THI, thiamin biosynthesis; GLU, gluconeogenesis.

Nevertheless, this analysis result might be classified further. In particular, some of the new targets are already well described in literature (see references in Table 5) and published evidence (e.g., essential gene) supports them as promising targets. In those cases where such validating evidence exists, the entry was marked referring to the literature listed in the table legend. Further testing of those already known genes should next focus on identifying lead compounds to target them. The remaining genes were considered regarding their suitability as potential candidates by affiliation to any important metabolic pathway and supporting biological knowledge. The genes that preserved thus their potential are marked by a colored box in column ("genetic test worth doing"). Yellow labeled entries denote potential candidates, while blue labeled entries are candidates that currently are under our experimental evaluation (e.g., AFUA_3G09290). Furthermore, blue boxes containing a black dot indicates candidates studied with a conditional expression system in the course of our experimental evaluation (AFUA_1G06940, AFUA_2G10520, AFUA_6G04700, AFUA_5G13130). Moreover, two of those genes could already be verified regarding their importance for fungal in vivo growth and can be considered as promising potential drug targets (Sasse et al., 2016).

We hence see in Table 5 several potential targets, many being validated in literature: specifically some proteins connected to amino acid biosynthesis and fatty acid biosynthesis are already confirmed as good targets and well-studied (UaZ, AFUA_2G06230; AroC, AFUA_5G13130; FasA, AFUA_3G04210; FasB, AFUA_3G04220). Recent results validated our predictions regarding targets of the shikimate pathway (Sasse et al., 2016). The predicted and experimentally validated targets chorismate synthase (ARO2, AFUA_1G06940) and chorismate mutase (AroC, AFUA_5G13130) show a high potential as antimycotic. From our results we can even derive a more general strategy (see our Summary Table 5): Targeting specifically expanded protein families of the fungal pathogen 
that are connected to its metabolism. For A. fumigatus this is for instance a phosphate transporter like Pho88 (AFUA_5G01960), a protein participating in inorganic phosphate acquisition. In fact, Pho88 was already studied in A. nidulans as well as in A. fumigatus (de Gouvêa et al., 2008). Similarly, other branches of primary metabolism contain promising targets (Table 5), for instance another metabolic target would be to disrupt phospholipid biosynthesis by targeting genes like cho1 (AFUA_4G13680) (Wolf et al., 2015).

AFUA_3G12320 lipase/serine esterase is another promising potential drug target. It has been studied in yeast only and it has no human homologs. Yeast homolog Lpl1 is nonessential and exerts phospholipase B activity to play a vital role in LD (lipid droplet) morphology, so its absence results in altered LD size (Selvaraju et al., 2014). However, as there is no auxotrophy and there might be redundancy with additional phospholipase $B$ genes. However, from a drug point of view, a phospholipase B blocker would be considered interesting, as a drug, because it would probably block all phospholipase B activities in general, resulting in an inviable phenotype.

In contrast, the vacuolar transporter chaperone (Vtc4, AFUA_2G09040) is a vacuolar membrane polyphosphate polymerase; from Candida we know that this is a putative polyphosphate synthetase with decreased expression in hyphae compared to yeast-form cells; moreover, it is a fungal-specific protein (no human or murine homolog) with a typical virulencefactor like expression during infection. Most components of the pathway, except this protein, are shared in all organisms; yet, this gene itself is an essential gene target and might be explored further.

Furthermore, some members of the phospholipid biosynthesis like cho1 (AFUA_4G13680), cho2 (AFUA_2G15970), and choC (AFUA_1G09050) (Tao et al., 2010) are also worthwhile testing, in particular as ChoC has no human homolog. The deletion of choC in A. nidulans was studied by Tao et al. (2010): It results in highly restricted vegetative growth, swelling at the hyphal tips and the complete blockage of asexual and sexual development on culture medium lacking choline. If we extrapolate to the clinic, this may be a promising block against various A. fumigatus infection routes. However, in the lung, surfactant may help to counter balance such an antimycotic, as dipalmitoyl phosphatidylcholine (aka: lecithin) is a major component of pulmonary surfactant, and may provide an alternative source of phospholipids.

Finally, for this phospholipid pathway a promising, not yet explored strategy would be to target the connected enzyme flippase. Phosphatidylserine(s) are actively held facing the cytosolic (inner) side of the cell membrane by the enzyme. Specific blocking of flippase (including known A. fumigatus variants, e.g., Z5, DRS2 flippase) should inhibit A. fumigatus growth.

\section{DISCUSSION}

The strategy to target metabolism in fungal pathogens has been advocated previously (e.g., Tao et al., 2010; Sasse et al., 2016) while the combination of different omics strategies for antimycotic pipelines can provide efficiently novel targets in these pathways. The three approaches we combined are each whole fields of their own: metabolic modeling, transcriptome analysis and the study of PPI are here combined in a specific and novel way to systematically identify targets of the human-pathogenic mold A. fumigatus. Related work includes a detailed metabolic analysis (Li et al., 2013) to improve itaconic acid production in different Aspergillus species, and a first study of essential genes according to flux models in A. fumigatus (Thykaer et al., 2009). Regarding the latter, we present here our own detailed model on primary metabolism and are much stricter in validating potential targets by combining several approaches as well as testing and including direct genetic evidence and detailed transcriptome data. There are several exciting transcriptome studies in $A$. fumigatus, in particular studies identifying invasion-related gene expression changes (McDonagh et al., 2008; Willger et al., 2008; Schrettl et al., 2010) and detailed analyses of PPI in fungal infections (Lamoth et al., 2015).

The key to success in the identification of new antimycotics is efficient implementation. For the latter this paper provides a broad overview on available approaches, as three different pipelines are combined and evaluated against each other. We are not dogmatic about any of these approaches, for instance, starting from our calculations given (see Supplementary Material) the metabolic approach can be pushed much further to identify new pathways ( $\mathrm{Li}$ et al., 2013), choke modes (Rahman and Schomburg, 2006), hub enzymes (Thadakamalla et al., 2005) and so on as visible in related work. The same applies for the transcriptome analysis (analyzing more and more different datasets). A nice example is the analysis of gliotoxin production and attenuation in A. fumigatus (O'Keeffe et al., 2014). Analysis of PPI networks in A. fumigatus is particularly topical (Lamoth et al., 2015; Remmele et al., 2015). Hence, our interactome pipeline can be further refined profiting from new interaction data-sets which constantly appear online and in the literature. We hence suggest here that each of these pipelines has high potential in antimycotic target search, however, they are virtually complementary strategies to identify key metabolic targets and that is the underlying rationale why we combined them.

Nevertheless, there are some inherent limitations. To show that the different pipelines work and deliver, we were rather conservative and strict in our overall criteria: anything being homologous to humans (even low homology) was discarded as a potential drug target, only central metabolism was targeted and so on. Such an approach is good for delivering certified, strong targets and can be used to validate the different pipelines by knowledge (see Section Drug Targets with Orthology to Functionally Important Genes of the Results part). However, as the results confirm, this yields also several targets that have already been identified or are currently tested.

Hence, this has to be extended in future by more subtle approaches. A first measure will be to loosen the strict requirement of absent orthology to human proteins, allowing distant homologs. Furthermore, primary metabolism is defined by its connection and requirement for cellular growth (reason to pick it for antimycotic design), however, it needs not to be central in the metabolic web. In particular further areas connected to cell 
wall metabolism, virulence or, for instance, iron utilization can and will be scrutinized further. Finally, the metabolic calculations in particular, but also interaction screens and transcriptomics allow more refined screening methods: this includes synthetic lethality (hitting two targets that together stop fungal growth, calculation for instance by metabolic flux analysis), and exerting the full power of the transcriptomic approach by looking at ten or more conditions to identify all metabolic genes that are at least expressed under some specific condition. Furthermore, there can be better targeting of auxotrophic mutants, for instance by knowledge-based approaches and creating toxic metabolic intermediates which stop fungal growth in the fungus without harming the human patient. We believe that these more sophisticated approaches will allow an even better view on new antifungal strategies against $A$. fumigatus infection.

\section{CONCLUSIONS}

Metabolism is interesting to target by novel antimycotic substances. Three different pipelines are made available here to investigate A. fumigatus in this respect. They validate potential targets from metabolism according to their importance in flux control and metabolic pathways, as key regulated enzymes under different challenging conditions or as central metabolic hubs with known protein structure, different from man, and, if possible, with some drug to target it. Together, these produced important targets, as validated by previous publications and currently ongoing experimental tests for about half of these. The others are made public here to allow further research and investigations. However, we followed a conservative approach demanding no homology to human proteins and avoiding more complex considerations such as targeting the same pathway twice or producing toxic intermediates. Future, more detailed analyses will follow up also these more sophisticated options. Additionally, the software and criteria applied can (and will) next be applied to other parts of the metabolic map in A. fumigatus

\section{REFERENCES}

Al-Bader, N., Vanier, G., Liu, H., Gravelat, F. N., Urb, M., Hoareau, C. M.Q., et al. (2010). Role of trehalose biosynthesis in Aspergillus fumigatus development, stress response, and virulence. Infect. Immun. 78, 3007-3018. doi: 10.1128/IAI.00813-09

Altschul, S. F., Madden, T. L., Schäffer, A. A., Zhang, J., Zhang, Z., Miller, W., et al. (1997). Gapped BLAST and PSI-BLAST: a new generation of protein database search programs. Nucleic Acids Res. 25, 3389-3402.

Andersen, M. R., Nielsen, M. L., and Nielsen, J. (2008). Metabolic model integration of the bibliome, genome, metabolome and reactome of Aspergillus niger. Mol. Syst. Biol. 4:178. doi: 10.1038/msb.2008.12

Bairoch, A. (2000). The ENZYME database in 2000. Nucleic Acids Res. 28, 304-305. doi: 10.1093/nar/28.1.304

Barrett, T., Wilhite, S. E., Ledoux, P., Evangelista, C., Kim, I. F., Tomashevsky, M., et al. (2013). NCBI GEO: archive for functional genomics data sets - Update. Nucleic Acids Res. 41, D991-D995. doi: 10.1093/nar/gks1193

Bertuzzi, M., Schrettl, M., Alcazar-Fuoli, L., Cairns, T. C., Muñoz, A., Walker, L. A., et al. (2014). The $\mathrm{pH}$-responsive PacC transcription factor of Aspergillus fumigatus governs epithelial entry and tissue invasion during pulmonary aspergillosis. PLoS Pathog. 10:e1004413. doi: 10.1371/journal.ppat.1004413 and can also handle any other organism of interest if protein sequences are sufficiently comprehensively known.

\section{AUTHOR CONTRIBUTIONS}

MK analyzed and calculated transcriptome and enzyme regulation data. MS and SG analyzed and calculated interactome-based data. CL calculated and analyzed metabolic fluxes and elementary modes. $\mathrm{HH}, \mathrm{NO}$, and $\mathrm{SK}$ and their $\mathrm{PhD}$ students $\mathrm{AD}, \mathrm{ZM}$, and $\mathrm{JB}$, respectively, provided A. fumigatus expertise and experimental expertise on all protein targets suggested. TD analyzed data, lead and guided the study. MK and TD drafted and finalized the manuscript, all authors contributed expert advice, gave own comments, and agreed to the final submitted version of the manuscript.

\section{FUNDING}

This study was supported by the German Federal Ministry of Education and Research (FKZ 031A408B to TD and 031A408A to SK), the Austrian Science Fund (FWF grant I1616 to $\mathrm{HH}$ ), and the Israel Ministry of Health (MOH 3-0000-11080 to NO) by funding the AspMetNet consortium during the first call of the Infect-ERA research co-ordination action.

\section{ACKNOWLEDGMENTS}

We thank George Leigh for native speaker corrections and stylistic suggestions. This publication was supported by the Open Access Publication Fund of the University of Würzburg.

\section{SUPPLEMENTARY MATERIAL}

The Supplementary Material for this article can be found online at: http://journal.frontiersin.org/article/10.3389/fmolb. 2016.00022

Brock, M. (2005). Generation and phenotypic characterization of Aspergillus nidulans methylisocitrate lyase deletion mutants: methylisocitrate inhibits growth and conidiation. Appl. Environ. Microbiol. 71, 5465-5475. doi: 10.1128/AEM.71.9.5465-5475.2005

Bruns, S., Seidler, M., Albrecht, D., Salvenmoser, S., Remme, N., Hertweck, C., et al. (2010). Functional genomic profiling of Aspergillus fumigatus biofilm reveals enhanced production of the mycotoxin gliotoxin. Proteomics 10, 3097-3107. doi: 10.1002/pmic.201000129

Caspi, R., Billington, R., Ferrer, L., Foerster, H., Fulcher, C. A., Keseler, I. M., et al. (2016). The MetaCyc database of metabolic pathways and enzymes and the BioCyc collection of pathway/genome databases. Nucleic Acids Res. 4, D471-D480. doi: 10.1093/nar/gkv1164

Cecil, A., Ohlsen, K., Menzel, T., François, P., Schrenzel, J., Fischer, A., et al. (2015). Modelling antimycotic and cytotoxic isoquinoline effects in Staphylococcus aureus, Staphylococcus epidermidis and mammalian cells. Int. J. Med. Microbiol. 305, 96-109. doi: 10.1016/j.ijmm.2014.11.006

Cecil, A., Rikanoviæ, C., Ohlsen, K., Liang, C., Bernhardt, J., Oelschlaeger, T. A., et al. (2011). Modeling antimycotic and cytotoxic effects of the dimeric isoquinoline IQ-143 on metabolism and its regulation in Staphylococcus aureus, Staphylococcus epidermidis and human cells. Genome Biol. 12:R24. doi: 10.1186/gb-2011-12-3-r24 
Conesa, A., and Götz, S. (2008). Blast2GO: a comprehensive suite for functional analysis in plant genomics. Int. J. Plant Genomics 2008:619832. doi: $10.1155 / 2008 / 619832$

Dagenais, T. R. T., and Keller, N. P. (2009). Pathogenesis of Aspergillus fumigatus in invasive aspergillosis. Clin. Microbiol. Rev. 22, 447-465. doi: 10.1128/CMR.00055-08

David, H., Özçelik, I. S., Hofmann, G., and Nielsen, J. (2008). Analysis of Aspergillus nidulans metabolism at the genome-scale. BMC Genomics 9:163. doi: $10.1186 / 1471-2164-9-163$

Davis, S., and Meltzer, P. S. (2007). GEOquery: a bridge between the Gene Expression Omnibus (GEO) and bioconductor. Bioinformatics 23, 1846-1847. doi: 10.1093/bioinformatics/btm254

de Gouvêa, P. F., Soriani, F. M., Malavazi, I., Savoldi, M., Goldman, M. H., Loss, O., et al. (2008). Functional characterization of the Aspergillus fumigatus PHO80 homologue. Fungal Genet. Biol. 45, 1135-1146. doi: 10.1016/j.fgb.2008.04.001

Denning, D. W. (1998). Invasive aspergillosis. Clin. Infect. Dis. 26, 781-803.

Dichtl, K., Samantaray, S., Aimanianda, V., Zhu, Z., Prévost, M.-C., Latgé, J.-P., et al. (2015). Aspergillus fumigatus devoid of cell wall $\beta$-1,3-glucan is viable, massively sheds galactomannan and is killed by septum formation inhibitors. Mol. Microbiol. 95, 458-471. doi: 10.1111/mmi.12877

Edgar, R., Domrachev, M., and Lash, A. E. (2002). Gene expression omnibus: NCBI gene expression and hybridization array data repository. Nucleic Acids Res. 30, 207-210. doi: 10.1093/nar/30.1.207

Edwards, R. A., Keller, L. H., and Schifferli, D. M. (1998). Improved allelic exchange vectors and their use to analyze 987P fimbria gene expression. Gene 207, 149-57.

Fischer, S., Brunk, B. P., Chen, F., Gao, X., Harb, O. S., Iodice, J. B., et al. (2011). Using OrthoMCL to assign proteins to OrthoMCL-DB groups or to cluster proteomes into new ortholog groups. Curr. Protoc. Bioinform. Chapter 6; Unit 6.12.1-19. doi: 10.1002/0471250953.bi0612s35

Huber, W., Carey, V. J., Gentleman, R., Anders, S., Carlson, M., Carvalho, B. S., et al. (2015). Orchestrating high-throughput genomic analysis with bioconductor. Nat. Methods 12, 115-121. doi: 10.1038/nmeth.3252

Kanehisa, M., and Goto, S. (2000). KEGG: kyoto encyclopedia of genes and genomes. Nucleic Acids Res. 28, 27-30. doi: 10.1093/nar/28.1.27

Kanehisa, M., Goto, S., Sato, Y., Kawashima, M., Furumichi, M., and Tanabe, M. (2014). Data, information, knowledge and principle: back to metabolism in KEGG. Nucleic Acids Res. 42, D199-D205. doi: 10.1093/nar/gkt1076

Kim, Y., Min, B., and Yi, G.-S. (2012). IDDI: integrated domain-domain interaction and protein interaction analysis system. Proteome Sci. 10:S9. doi: 10.1186/1477-5956-10-S1-S9

Kohanski, M. A., Dwyer, D. J., and Collins, J. J. (2010). How antimycotics kill bacteria: from targets to networks. Nat. Rev. Microbiol. 8, 423-435. doi: 10.1038/nrmicro2333

Krappmann, S., Helmstaedt, K., Gerstberger, T., Eckert, S., Hoffmann, B., Hoppert, M., et al. (1999). The aroC gene of Aspergillus nidulans codes for a monofunctional, allosterically regulated chorismate mutase. J. Biol. Chem. 274, 22275-22282.

Lamoth, F., Juvvadi, P. R., Soderblom, E. J., Moseley, M. A., and Steinbach, W. J. (2015). Hsp70 and the cochaperone StiA (Hop) Orchestrate Hsp90-mediated caspofungin tolerance in Aspergillus fumigatus. Antimicrob. Agents Chemother. 59, 4727-4733. doi: 10.1128/AAC.00946-15

Li, A., Caspers, M., and Punt, P. (2013). A systems biology approach for the identification of target genes for the improvement of itaconic acid production in Aspergillus species. BMC Res. Notes 6:505. doi: 10.1186/1756-0500-6-505

Li, L., Stoeckert, C. J. Jr., and Roos, D. S. (2003). OrthoMCL: identification of ortholog groups for eukaryotic genomes. Genome Res. 13, 2178-2189. doi: $10.1101 /$ gr. 1224503

Liang, C., Liebeke, M., Schwarz, R., Zühlke, D., Fuchs, S., Menschner, L., et al. (2011). Staphylococcus aureus physiological growth limitations: insights from flux calculations built on proteomics and external metabolite data. Proteomics 11, 1915-1935. doi: 10.1002/pmic.201000151

Lin, H.-N., Chen, C.-T., Sung, T.-Y., Ho, S.-Y., and Hsu, W.-L. (2009). Protein subcellular localization prediction of eukaryotes using a knowledge-based approach. BMC Bioinform. 10(Suppl. 1):S8. doi: 10.1186/1471-2105-10-S15-S8

Luo, H., Lin, Y., Gao, F., Zhang, C.-T., and Zhang, R. (2013). DEG 10, an update of the database of essential genes that includes both protein-coding genes and noncoding genomic elements. Nucleic Acids Res. 42, D574-D580. doi: 10.1093/nar/gkt1131

Luo, Q., Pagel, P., Vilne, B., and Frishman, D. (2011). DIMA 3.0: domain Interaction Map. Nucleic Acids Res. 39, D724-D729. doi: 10.1093/nar/gkq1200

Ma, Y., Qiao, J., Liu, W., Wan, Z., Wang, X., Calderone, R., et al. (2008). The sho1 sensor regulates growth, morphology, and oxidant adaptation in Aspergillus fumigatus but is not essential for development of invasive pulmonary aspergillosis. Infect. Immun. 76, 1695-1701. doi: 10.1128/IAI.01507-07

Marx, H., Mattanovich, D., and Sauer, M. (2008). Overexpression of the riboflavin biosynthetic pathway in Pichia pastoris. Microb. Cell Fact. 7:23. doi: 10.1186/1475-2859-7-23

McDonagh, A., Fedorova, N. D., Crabtree, J., Yu, Y., Kim, S., Chen, D., et al. (2008). Sub-telomere directed gene expression during initiation of invasive aspergillosis. PLoS Pathog. 4:e1000154. doi: 10.1371/journal.ppat.10 00154

Michal, G., and Schomburg, D. (2012). Biochemical Pathways: An Atlas of Biochemistry and Molecular Biology. New York, NY: John Wiley \& Sons.

Mouyna, I., Morelle, W., Vai, M., Monod, M., Léchenne, B., Fontaine, T., et al. (2005). Deletion of GEL2 encoding for a beta(1-3)glucanosyltransferase affects morphogenesis and virulence in Aspergillus fumigatus. Mol. Microbiol. 56, 1675-1688. doi: 10.1111/j.1365-2958.2005.04654.x

Muszkieta, L., Beauvais, A., Pähtz, V., Gibbons, J. G., Anton Leberre, V., Beau, R., et al. (2013). Investigation of Aspergillus fumigatus biofilm formation by various "omics" approaches. Front. Microbiol. 4:13. doi: 10.3389/fmicb.2013.00013

Oestreicher, N., and Scazzocchio, C. (1993). Sequence, regulation, and mutational analysis of the gene encoding urate oxidase in Aspergillus nidulans. J. Biol. Chem. 268, 23382-23389.

Oestreicher, N., Sealy-Lewis, H. M., and Scazzocchio, C. (1993). Characterisation, cloning and integrative properties of the gene encoding urate oxidase in Aspergillus nidulans. Gene 132, 185-192.

O'Keeffe, G., Hammel, S., Owens, R. A., Keane, T. M., Fitzpatrick, D. A., Jones, G. W., et al. (2014). RNA-seq reveals the pan-transcriptomic impact of attenuating the gliotoxin self-protection mechanism in Aspergillus fumigatus. BMC Genomics 15:894. doi: 10.1186/1471-2164-15-894

Puttikamonkul, S., Willger, S. D., Grahl, N., Perfect, J. R., Movahed, N., Bothner, B., et al. (2010). Trehalose 6-phosphate phosphatase is required for cell wall integrity and fungal virulence but not trehalose biosynthesis in the human fungal pathogen Aspergillus fumigatus. Mol. Microbiol. 77, 891-911. doi: 10.1111/j.1365-2958.2010.07254.x

Rahman, S. A., and Schomburg, D. (2006). Observing local and global properties of metabolic pathways: "load points" and "choke points" in the metabolic networks. Bioinformatics 22, 1767-1774. doi: 10.1093/bioinformatics/btl181

R Core Team (2015). R: A Language and Environment for Statistical Computing. Vienna: R Foundation for Statistical Computing. Available online at: http:// www.r-project.org/

Remmele, C. W., Luther, C. H., Balkenhol, J., Dandekar, T., Müller, T., and Dittrich, M. T. (2015). Integrated inference and evaluation of host-fungi interaction networks. Front. Microbiol. 6:764. doi: 10.3389/fmicb.2015.00764

Richie, D. L., Miley, M. D., Bhabhra, R., Robson, G. D., Rhodes, J. C., and Askew, D. S. (2007). The Aspergillus fumigatus metacaspases CasA and CasB facilitate growth under conditions of endoplasmic reticulum stress. Mol. Microbiol. 63, 591-604. doi: 10.1111/j.1365-2958.2006.05534.x

Ritchie, M. E., Phipson, B., Wu, D., Hu, Y., Law, C. W., Shi, W., et al. (2015). limma powers differential expression analyses for RNA-sequencing and microarray studies. Nucleic Acids Res. 43, e47. doi: 10.1093/nar/gkv007

Ruijter, G. J. G., Bax, M., Patel, H., Flitter, S. J., van de Vondervoort, P. J. I., de Vries, R. P., et al. (2003). Mannitol is required for stress tolerance in Aspergillus niger conidiospores. Eukaryot. Cell 2, 690-698. doi: 10.1128/EC.2.4.690-698.2003

Salwinski, L., Miller, C. S., Smith, A. J., Pettit, F. K., Bowie, J. U., and Eisenberg, D. (2004). The database of interacting proteins: 2004 update. Nucleic Acids Res. 32, D449-D451. doi: 10.1093/nar/gkh086

Sasse, A., Hamer, S. N., Amich, J., Binder, J., and Krappmann, S. (2016). Mutant characterisation and in vivo conditional repression identify aromatic amino acid biosynthesis to be essential for Aspergillus fumigatus virulence. Virulence 7, 56-62. doi: 10.1080/21505594.2015.1109766

Schrettl, M., Beckmann, N., Varga, J., Heinekamp, T., Jacobsen, I. D., Jöchl, C., et al. (2010). HapX-mediated adaption to iron starvation is crucial 
for virulence of Aspergillus fumigatus. PLoS Pathog. 6:e1001124. doi: 10.1371/journal.ppat.1001124

Schwarz, R., Liang, C., Kaleta, C., Kühnel, M., Hoffmann, E., Kuznetsov, S., et al. (2007). Integrated network reconstruction, visualization and analysis using YANAsquare. BMC Bioinform. 8:313. doi: 10.1186/1471-2105-8-313

Schwarz, R., Musch, P., von Kamp, A., Engels, B., Schirmer, H., Schuster, S., et al. (2005). YANA - a software tool for analyzing flux modes, gene-expression and enzyme activities. BMC Bioinform. 6:135. doi: 10.1186/1471-2105-6-135

Selvaraju, K., Rajakumar, S., and Nachiappan, V. (2014). Identification of a phospholipase B encoded by the LPL1 gene in Saccharomyces cerevisiae. Biochim. Biophys. Acta 1842, 1383-1392. doi: 10.1016/j.bbalip.2014.06.013

Shi, L. N., Li, F., Huang, M., Lu, J., Kong, X., Wang, S., et al. (2012). Immunoproteomics based identification of thioredoxin reductase GliT and novel Aspergillus fumigatus antigens for serologic diagnosis of invasive aspergillosis. BMC Microbiol. 12:11. doi: 10.1186/1471-2180-12-11

Shityakov, S., Dandekar, T., and Förster, C. (2015). Gene expression profiles and protein-protein interaction network analysis in AIDS patients with HIVassociated encephalitis and dementia. HIV AIDS (Auckl). 7, 265-276. doi: 10.2147/HIV.S88438

Smoot, M. E., Ono, K., Ruscheinski, J., Wang, P. L., and Ideker, T. (2011). Cytoscape 2.8: new features for data integration and network visualization. Bioinformatics 27, 431-432. doi: 10.1093/bioinformatics/btq675

Tao, L., Gao, N., Chen, S., and Yu, J.-H. (2010). The choC gene encoding a putative phospholipid methyltransferase is essential for growth and development in Aspergillus nidulans. Curr. Genet. 56, 283-296. doi: 10.1007/s00294-010-0300-8

Thadakamalla, H. P., Albert, R., and Kumara, S. R. (2005). Search in weighted complex networks. Phys. Rev. E Stat. Nonlin. Soft Matter Phys. $72(6 \mathrm{Pt}$ 2):066128. doi: 10.1103/PhysRevE.72.066128

Thykaer, J., Andersen, M. R., and Baker, S. E. (2009). Essential pathway identification: from in silico analysis to potential antifungal targets in Aspergillus fumigatus. Med. Mycol. 47(Suppl. 1), S80-S87. doi: $10.1080 / 13693780802455305$

Toomey, D., Hoppe, H. C., Brennan, M. P., Nolan, K. B., and Chubb, A. J. (2009). Genomes2Drugs: identifies target proteins and lead drugs from proteome data. PLoS ONE 4:e6195. doi: 10.1371/journal.pone.0006195
Valerius, O., Draht, O., Kübler, E., Adler, K., Hoffmann, B., and Braus, G. H. (2001). Regulation of hisHF transcription of Aspergillus nidulans by adenine and amino acid limitation. Fungal Genet. Biol. 32, 21-31. doi: 10.1006/fgbi.2000. 1244

Vongsangnak, W., Olsen, P., Hansen, K., Krogsgaard, S., and Nielsen, J. (2008). Improved annotation through genome-scale metabolic modeling of Aspergillus oryzae. BMC Genomics 9:245. doi: 10.1186/1471-2164-9-245

von Kamp, A., and Schuster, S. (2006). Metatool 5.0: fast and flexible elementary modes analysis. Bioinformatics 22, 1930-1931. doi: 10.1093/bioinformatics/btl267

Willger, S. D., Puttikamonkul, S., Kim, K.-H., Burritt, J. B., Grahl, N., Metzler, L. J., et al. (2008). A sterol-regulatory element binding protein is required for cell polarity, hypoxia adaptation, azole drug resistance, and virulence in Aspergillus fumigatus. PLoS Pathog. 4:e1000200. doi: 10.1371/journal.ppat. 1000200

Wolf, J. M., Espadas, J., Luque-Garcia, J., Reynolds, T., and Casadevall, A. (2015). Lipid biosynthetic genes affect Candida albicans Extracellular vesicle morphology, cargo, and immunostimulatory properties. Eukaryot. Cell 14, 745-754. doi: 10.1128/EC.00054-15

Yellaboina, S., Tasneem, A., Zaykin, D. V., Raghavachari, B., and Jothi, R. (2011). DOMINE: a comprehensive collection of known and predicted domain-domain interactions. Nucleic Acids Res. 39, D730-D735. doi 10.1093/nar/gkq1229

Conflict of Interest Statement: The authors declare that the research was conducted in the absence of any commercial or financial relationships that could be construed as a potential conflict of interest.

Copyright (๑ 2016 Kaltdorf, Srivastava, Gupta, Liang, Binder, Dietl, Meir, Haas, Osherov, Krappmann and Dandekar. This is an open-access article distributed under the terms of the Creative Commons Attribution License (CC BY). The use, distribution or reproduction in other forums is permitted, provided the original author(s) or licensor are credited and that the original publication in this journal is cited, in accordance with accepted academic practice. No use, distribution or reproduction is permitted which does not comply with these terms. 